\title{
A two-process theory of the response to size and distance
}

\author{
WALTER C. GOGEL \\ University of California, Santa Barbara, California \\ and \\ JOSÉ APARECIDO DA SILVA \\ University of São Paulo, Ribeirão Preto, Brazil
}

\begin{abstract}
Evidence for a two-process theory of the observer's visual judgment of size and distance is summarized and evaluated. The primary process, as expressed by the size-distance invariance hypothesis (SDIH), concerns the interrelation of perceived size $\left(S^{\prime}\right)$ and perceived distance $\left(D^{\prime}\right)$, independent of information derived from memory or suggestion. The secondary process involves information regarding size (from memory or suggestion) not contained in the immediate visual stimulus. If the primary and secondary sources of size information differ, the object is seen as off-sized. This off-sized perception can be used to modify the direct report of the distance of the object. In the case of the casual suggestion of size, the off-sized judgment produces a nonperceptual (cognitive) report of distance (Gogel, 1981b). In the case of the memory of the size of a familiar object, some of the effect on the direct report of perceived distance is perceptual, but most is cognitive (Gogel, 1976). In the case of optical expansion, the effect of off-sized judgments on distance responses produces a clear modification of perceived distance (Swanston \& Gogel, 1986). The experimental distinction between primary and secondary processes is facilitated by comparing measures of perceived distance obtained from direct procedures (e.g., verbal reports of distance) with those obtained from indirect procedures (e.g., the head motion procedure). The former (direct) methods reflect both primary and secondary sources of information. The latter (indirect) methods are sensitive only to primary sources. The significance of the two-process theory for the specification of the SDIH and the explanation of other visual phenomena is discussed.
\end{abstract}

In this paper we consider the evidence for a two-process theory of the observer's response to visual information regarding size and distance. The primary process is responsible for the interrelation of perceived size and perceived distance, consistent with the size-distance invariance hypothesis (SDIH) (Epstein, Park, \& Casey, 1961; Gilinsky, 1951; Kilpatrick \& Ittelson, 1953; Schlosberg, 1950). The primary process utilizes sensory information available in the immediate visual stimulus. It does not require a memory of prior spatial extents, and it invariably results in perceptions. An example of the primary process is the perception of size (for a given size on the retina) resulting from a perception of distance as determined by oculomotor cues and binocular disparity. The secondary process is based on the primary process. The secondary process is representational, or cognitive, in that it involves a memory or expectation of size resulting from exposure to previous stimuli or from suggestion. This secondary process does not always or even usually result in a modifi-

The preparation of this paper was supported by United States Public Health Service Grant MH39457 from the National Institute of Mental Health to Walter C. Gogel and by Grants 82/0033-6 and 84/1632-6 from the Fundação de Amparo a Pesquisa do Estado de São Paulo (FAPESP), Brazil, to José Aparecido Da Silva. Address correspondence to Walter C. Gogel, Department of Psychology, University of California, Santa Barbara, CA 93106. cation of perceptions, although it has been found recently that, under proper conditions, its effect on the response to distance can be clearly perceptual (Swanston \& Gogel, 1986). As will be discussed, these two processes have been distinguished experimentally; the results are relevant to the basic problem of how and under what conditions information not available in the immediate stimulus, but available from memory or suggestion, can modify what is perceived.

\section{THE PROBLEM}

The need to postulate more than one process in the response to size and distance information is evident in research concerned with the effect of suggestion on reports of size and distance. This research has been conducted within the paradigm of the SDIH, which in its usual form is that

$$
S^{\prime} / D^{\prime}=S / D=\tan \theta,
$$

where $S^{\prime}$ is the perceived and $S$ is the physical size of the stimulus, $D^{\prime}$ is the perceived and $D$ is the physical distance of the stimulus from the observer, and $\theta$ is the visual angle subtended by the stimulus at the nodal point of the eye. ${ }^{1}$ The usual procedure for investigating the role of suggestion on the response to size and distance is to pro- 
vide a suggestion as to the size of the object and, with $D$ constant, to determine the effect of this suggestion on $D^{\prime}$ in Equation 1. For example, in a study by Hastorf (1950), observers were presented with a monocularly viewed object whose size could be given more than one interpretation. One stimulus (a circular object) was identified to the observer by the experimenter as either a billiard ball or a ping-pong ball, thus presumably specifying $S^{\prime}$. The observer's task was to adjust the object in apparent distance (by adjusting its physical size) until it appeared to be at a designated distance, as measured using a comparison alley containing a variety of distance cues. Since the physical distance of the object was constant and its perceived size was presumably specified by the identification, varying its physical size modified $\theta$ and thus presumably $D^{\prime}$ in Equation 1 . It was found that the physical size adjusted to achieve the same apparent distance in the comparison alley was greater for the larger suggested size. It seems from this result that a suggestion (identification) from the experimenter as to the size of an object, otherwise ambiguous in its representational size, can modify perceived size and hence perceived distance. If correct, this conclusion is of great theoretical importance, because it indicates that perception can be readily modified by suggestion. One purpose of this paper is to examine the validity of this conclusion.

A number of additional studies indicate that suggestions regarding size can, to some degree at least, change perceived size, as concluded by the experimenter from the suggestions' effect on reported distance (Equation 1). Methods of providing suggestions regarding size include giving verbal or tactual information as to the size of the target (Coltheart, 1969, 1970; Park \& Michaelson, 1974), giving the target the name of a familiar object (Baird, 1963; Park \& Michaelson, 1974), or providing a familiar object or familiar configuration as the target to be judged (e.g., Epstein, 1965; Eriksson \& Zetterberg, 1975; Fitzpatrick, Pasnak, \& Tyer, 1982; Gogel \& Mertens, 1967; Ittelson, 1951b; Park \& Michaelson, 1974). The last method, which is called the familiar-size cue to distance, has been found to be the most effective method for modifying reported distance, according to Park and Michaelson (1974).

It may seem reasonable that experience would permit the familiar size of a stimulus to determine its perceived size, and thus, as expected from Equation 1, to determine its perceived distance. It is more difficult, however, to accept the notion that casual suggestion, produced verbally or tactually or by alluding to familiar examples, is able to modify perceived size and thus to modify perceived distance. To accept such a conclusion is equivalent to asserting that perceived size is readily manipulated by information other than that contained in the immediate visual stimulus. This conclusion is compelling, however, only if it is assumed in the above studies that a direct response to distance obtained by, for example, verbal reports of distance necessarily provides a valid measure of perceived distance, which in turn would provide evidence that the memory or suggestion of size indeed modified perceived size. This assumption will be questioned in this review.

\section{OFF-SIZED PERCEPTIONS}

\section{The Theory of Off-Sized Perceptions}

It is possible that information regarding the size of a visible object provided by assumed size (resulting from past experience or suggestion) can modify a direct judgment of distance, such as a verbal report or a judgment of position with respect to a comparison alley, without having modified perceived size. How can this occur? Suppose that the remembered or suggested size (labeled $S_{\mathrm{c}}$ ) differs from the perceived size $\left(S^{\prime}\right)$ of the object as presented in the experiment. In this case the perceived size of the object, rather than being modified to correspond with the memory or suggestion, might remain unchanged, with the object then seen as nonnormal in size. This will be called an off-sized perception or off-sized judgment, defined as $S_{\mathrm{c}} / S^{\prime}$. If $S_{\mathrm{c}} / S^{\prime}>1$, the object is seen as a small off-sized object; if $S_{\mathrm{c}} / S^{\prime}<1$, the object is seen as a large off-sized object. Because one of the terms, $S_{\mathrm{c}}$, is representational and the other, $S^{\prime}$, is perceptual, it seems reasonable to call the response either an off-sized judgment or an off-sized perception, and both terms will be used in this discussion.

What is the probable effect of off-sized judgments on a direct report of distance? There is evidence (Carlson, 1960, 1977; Carlson \& Tassone, 1962) that observers expect an object to look smaller when it is at a far distance then when it is at a near distance. A likely reason for this expectation is that in visual fields extended in distance, cues to the distance of an object decrease in number and effectiveness as the physical distance of the object increases (Gogel, 1974). This reduction in the amount of available information as physical distance increases produces a perceptual underestimation of the distance of far, relative to near, objects. In agreement with Equation 1, this perceptual underestimation results in an object's appearing smaller when it is distant than when it is near the observer. Conversely, if the object appears larger or smaller than normal (an off-sized judgment), it often will be reported to be at a nearer or farther distance, respectively, than the distance at which it appears. Or, as expressed in equation form, an off-sized judgment $\left(S_{\mathrm{c}} / S^{\prime} \neq 1\right)$ results in distance information $\left(D_{\mathrm{c}}\right)$ different from perceived distance $\left(D^{\prime}\right)$, such that

$$
D_{\mathrm{c}}=D^{\prime}\left(S_{\mathrm{c}} / S^{\prime}\right) \text {. }
$$

The terms $S^{\prime}$ and $D^{\prime}$ are part of the primary process as expressed by the SDIH (Equation 1). The terms $S_{\mathrm{c}}$ and $D_{c}$ are part of the secondary process as expressed by Equation 2. According to Equation 2, the secondary process requires information $\left(S^{\prime}\right.$ and $\left.D^{\prime}\right)$ produced by the primary process, as well as the representational information involved in specifying $S_{\mathrm{c}}$. The term $S_{\mathrm{c}}$, whether or not it differs from $S^{\prime}$, can always be classified as cognitive, because it is not specified by the immediate stimulus but in- 
stead refers to some stored and retrieved (remembered) internal representation of the size of the object. Whether $D_{\mathrm{c}}$ is perceptual or cognitive is more difficult to specify. If $S_{\mathrm{c}}$ is produced by casual suggestion, $D_{\mathrm{c}}$ seems to be completely nonperceptual (Gogel, 1981). If $S_{\mathrm{c}}$ is produced by the starting size of an optical expansion, $D_{\mathrm{c}}$ can be clearly perceptual. When $S_{\mathrm{c}}=S^{\prime}, D_{\mathrm{c}}=D^{\prime}$, and both the primary and secondary (when available) processes contribute to the same response of size and distance. In situations in which $D_{\mathrm{c}}$ differs from $D^{\prime}$, the observer has two sources of conflicting information with which to determine the direct report of distance. Let $a$ be the weight given to $D^{\prime}$ and $1-a$ be the weight given to $D_{c}$ by the observer in the response $D_{R}$ to distance. Assuming a simple weighting function for $D_{\mathrm{R}}$,

$$
D_{\mathrm{R}}=a D^{\prime}+(1-a) D_{\mathrm{c}} .
$$

Similarly, if $b$ and $1-b$ are the weights given by the observer to $S^{\prime}$ and $S_{\mathrm{c}}$, in the direct report of size $\left(S_{\mathrm{R}}\right)$, the simple weighting function for $S_{R}$ is

$$
S_{\mathrm{R}}=b S^{\prime}+(1-b) S_{\mathrm{c}}
$$

(Gogel, 1976). It is likely that a number of circumstances will modify the weight the observer gives to $D^{\prime}$ relative to $D_{\mathrm{c}}$ or to $S^{\prime}$ relative to $S_{\mathrm{c}}$ in situations in which $S^{\prime} \neq$ $S_{\mathrm{c}}$ and, therefore, $D^{\prime} \neq D_{\mathrm{c}}$. For example, if many effective cues to distance are available, it is unlikely that presenting an observer with a half-sized playing card after presentation of a full-sized playing card at the same distance would elicit a verbal report that the perceived distance of the card has increased substantially (Predebon, Wenderoth, \& Curthoys, 1974). The more effective the sensory information, the more likely it is that $S^{\prime}$ and $D^{\prime}$ will be heavily weighted in a direct response to size and distance (Gogel \& Sturm, 1971). Instructions also might be expected to modify the weighting, determining a direct response to size. It seems likely, for example, that apparent instructions would increase the weight given to $S^{\prime}$ over that given to $S_{\mathrm{c}}$ in the direct report of size, whereas objective instructions emphasizing the need for the response to reflect physical size would increase the weight given to $S_{c}$. The terms $S_{c}$ and $S^{\prime}$ might be more easily disentangled by the observer than would the terms $D_{c}$ and $D^{\prime}$, so that modifying the weights given to $D_{\mathrm{c}}$ and $D^{\prime}$ by instructions might be difficult (Gogel \& Da Silva, 1986).

\section{Off-Sized Perceptions and the Egocentric Reference Distance}

In investigating the effect of assumed (familiar or suggested) size on the perception of size and distance, the experimenter often attempts to eliminate all cues to size and distance except that of assumed size. Thus, for example, Fitzpatrick et al. (1982) used, as a criterion for achieving the necessary reduction of extraneous cues to distance, the inability of the observer to discriminate the physical distance of objects in the absence of familiar-size cues. Unfortunately, this criterion does not guarantee that all factors other than familiarity that might determine the apparent distance of the objects from the observer (apparent egocentric distance) are absent. It is likely that some state of the oculomotor system is always available to determine a perception of egocentric distance. For example, a small restrictive viewing aperture can be used to eliminate the changes in accommodation that normally occur for objects at different distances. However, the accommodation then will be a constant resting value of about $67 \mathrm{~cm}$ (Hennessy, Iida, Shiina, \& Leibowitz, 1976; Owens \& Leibowitz, 1983), and this accommodative state can indicate to the observer that the stimulus is at that near distance, regardless of its actual distance from the observer. In addition, unless a small, dim point of light is the stimulus (Owens \& Leibowitz, 1983), the convergence of the eyes, despite monocular observation, will be determined by the resting state of accommodation (accommodative convergence) and will be available as a cue to distance. It is possible to eliminate all information as to the particular physical distance of an object. What is much more difficult is to eliminate all oculomotor responses that would indicate that the object is at some particular distance. Even if this were achieved, an additional factor, the specific distance tendency (SDT), would remain to determine a perceived egocentric distance for the object. The SDT is the tendency, in the absence of effective egocentric cues to physical distance, for an object to appear to be at a distance of several meters from the observer (Gogel, 1965). There is evidence that the SDT is indeed a perceptual tendency and not simply a default response in the absence of definitive distance cues (Gogel, 1969b; Gogel \& Tietz, 1973). Although it has been found that the SDT is correlated with the resting state of convergence (Owens \& Leibowitz, 1976), there is now evidence that these two factors are not identical (Gogel, 1982). The oculomotor states, including whatever other distance cues remain despite impoverished conditions of observation, together with the SDT, result in a composite distance factor that has been called the egocentric reference distance. This reference distance, under reduced cue conditions, is likely to result in a target's appearing to be a few meters from the observer, regardless of its physical distance. Thus, distance information, provided by the egocentric reference distance, is present even under the most stringent conditions of cue reduction (Gogel, 1972; Mershon \& Lembo, 1977). It follows that unless familiar size is quite effective as a cue to distance, the perceived egocentric distance of a familiar object, regardless of its actual distance, will deviate in the direction of the egocentric reference distance. If this happens, consistent with the SDIH (Equation 1), a normal-sized familiar object will be perceived as normal in size only when its physical distance coincides with the egocentric reference distance. Thus, to the degree that the egocentric reference distance differs from the physical distance, and to the degree that familiar size is ineffective as a cue to distance, under otherwise reduced conditions of observation, a normal-sized familiar object will be perceived as off-sized. If the physical distance of the familiar ob- 
ject is less than the egocentric reference distance, the familiar object will be seen as a large off-sized object $\left(S_{\mathrm{c}} / S^{\prime}<1\right)$. If the physical distance is greater than the egocentric reference distance, it will be seen as a small off-sized object $\left(S_{\mathrm{c}} / S^{\prime}>1\right)$.

\section{An Illustration of the Theory of Off-Sized Perceptions}

Figure 1 can be used to illustrate the discussion of primary and secondary processes in the direct response to size and distance. Two situations are shown, each involving a single presentation of a familiar object, illuminated in an otherwise totally dark surround and viewed monocularly. In the left drawing, a normal-sized playing card $\left(9 \mathrm{~cm}\right.$ high) is presented at a physical distance $\left(D_{\mathrm{n}}=\right.$ $1 \mathrm{~m})$ near the observer. In the right drawing, a normalsized chair $(76 \mathrm{~cm}$ high) is presented at a physical distance $\left(D_{\mathrm{f}}=4 \mathrm{~m}\right)$ more distant from the observer. The vertical panels identifying physical or perceived distances in the drawings are for illustrative purposes only and are not represented as actually present. To simplify the discussion, it is assumed that all cues to egocentric distance are absent except the possible cue of familiar size, and that this cue is completely ineffective. This leaves only the SDT (the specific distance is here assumed to be $2 \mathrm{~m}$ ) to determine the perceived distance of the familiar object. Consequently, when either of the familiar objects is presented, it will appear to be at the SDT distance of $2 \mathrm{~m}$ from the observer. Thus, when the playing card is presented, it will be perceived to be at twice its physical distance, and, in agreement with the SDIH, it will appear to be twice its normal height $\left(S^{\prime}=18 \mathrm{~cm}\right)$. When the chair is presented, it will be perceived at half its physical distance, and, in agreement with the SDIH, it will appear to be half its normal height $\left(S^{\prime}=38 \mathrm{~cm}\right)$.

According to Equation 2, the off-sized perceptions $\left(S_{c} / S^{\prime}\right)$ will provide cognitive information $\left(D_{c}\right)$ that the familiar objects are indeed at their physical distances. Thus, in the case of the playing card, $D_{\mathrm{c}}=2 \mathrm{~m}(1 / 2)=$ $1 \mathrm{~m}$ and, in the case of the chair, $D_{\mathrm{c}}=2 \mathrm{~m}(2)=4 \mathrm{~m}$. This effect of $D_{c}$, to correct the response resulting from errors in perceived distance, can also apply to more normal conditions. For example, because of the loss of distance cues at far distances, a horse at $\mathbf{4 0 0} \mathrm{m}$ may appear to be at $200 \mathrm{~m}$; therefore, consistent with the SDIH, it would appear to be only half its normal or expected size. According to Equation 2, the perception $\left(D^{\prime}\right)$ that the horse is at $200 \mathrm{~m}$ and appears half-sized $\left(S_{\mathrm{c}} / S^{\prime}=2\right)$ could result in a response $\left(D_{R}\right)$, based on $D_{c}$, that the horse is actually at $400 \mathrm{~m}$. In these examples, the secondary-

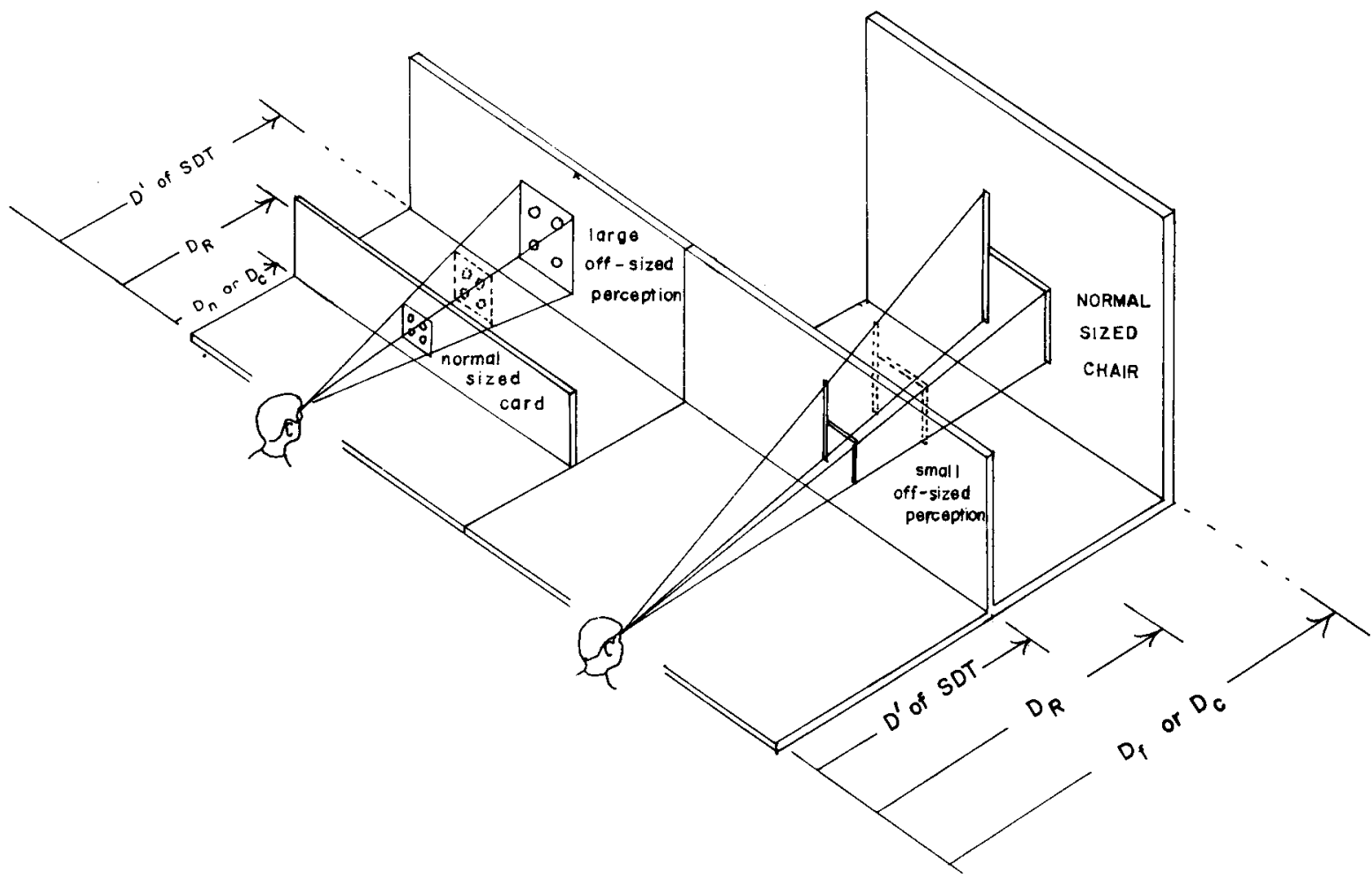

Figure 1. Illustration of the perception of familiar objects as off-sized as a consequence of perceiving them (erroneously) to be at a distance determined by the specific distance tendency ( $D^{\prime}$ of SDT). This perceptual error in distance need not completely determine the verbal report of distance. The observer can use the distance information $\left(D_{\mathrm{c}}\right)$ provided by $D^{\prime}$ and the off-sized perception $\left(S_{\mathrm{c}} / S^{\prime}\right)$, as indicated by Equation 2 (see text), to eliminate the error in the verbal report that would occur from $D^{\prime}$ alone. The distance $\left(D_{R}\right)$ and size $\left(S_{\mathrm{R}}\right)$ reported are often compromises between the $D_{\mathrm{c}}$ and $D^{\prime}$ values for distance and between the $S_{\mathrm{c}}$ and $S^{\prime}$ values for size. 
process $S_{\mathrm{c}}$ and $D_{\mathrm{c}}$ provides a nonperceptual (cognitive) correction for distance errors resulting from the primary process.

Figure 1 also can be used to illustrate the application of the weighting equations (Equations 3 and 4 ) to the two sources of information regarding size and distance. One source (the primary process) provides the information that the card (chair) is 18 (38) $\mathrm{cm}$ high and is at a distance of $2 \mathrm{~m}$. The other (the secondary process) informs the observer that the card (chair) is 9 (76) $\mathrm{cm}$ high (its normal size, $S_{\mathrm{c}}$ ) and is at a distance of 1 (4) $\mathrm{m}$ (its physical distance). Because the two contradictory sources of information are simultaneously available with each familiar object, the response as to the size or distance of the familiar object ( $S_{R}$ or $D_{R}$ ) will depend upon the value given by the observer to the weights $a$ (Equation 3) and $b$ (Equation 4). If $a=b=1$, only the values of the primary process will determine the responses. If $a=b$ $=0$, only the values of the secondary process will determine the responses. If $a=b=.5$, the primary and secondary processes will contribute equally to the responses as to size and distance. In the latter instance, given the $S^{\prime}, D^{\prime}, S_{\mathrm{c}}$, and $D_{\mathrm{c}}$ values cited in the above illustration, the verbal reports of size $\left(S_{\mathrm{R}}\right)$ and distance $\left(D_{\mathrm{R}}\right)$, according to Equations 3 and 4 , would be that the card was $11 / 2$ times its normal size at a distance of $1.5 \mathrm{~m}$ and that the chair was $3 / 4$ of its normal size at a distance of $3 \mathrm{~m}$. The dashed outlines of card and chair in Figure 1 illustrate this result. Thus, the experimenter would conclude from the verbal reports that familiar size is totally ineffective as a cue to distance in the first instance of weight assignment, that it is totally effective in the second instance, and only partially effective in the third.

\section{Invariance Functions}

If $D^{\prime} \tan \theta$ from Equation 1 is substituted for $S^{\prime}$ in Equation 2, it follows that

$$
S_{\mathrm{c}} / D_{\mathrm{c}}=S / D=\tan \theta .
$$

Equations 1 and 5 are similar in form but not in meaning. In Equation 1, $S^{\prime}$ and $D^{\prime}$ represent a primary process and are always perceptual. In Equation $5, S_{\mathrm{c}}$ is always representational (cognitive), and, as will be discussed, $D_{c}$, although often cognitive under some conditions, can be perceptual. As considered in relation to Figure 1 , if direct responses ( $S_{\mathrm{R}}$ and $\left.D_{\mathrm{R}}\right)$, such as verbal reports of size and distance, are obtained, it is not clear whether they are the result of primary or secondary processes or some combination of both. According to the weighting equations (Equations 3 and 4),

$$
S_{\mathrm{R}} / D_{\mathrm{R}}=\left[b S^{\prime}+(1-b) S_{\mathrm{c}}\right] /\left[a D^{\prime}+(1-a) D_{\mathrm{c}}\right] \text {. }
$$

For all cases in which $a=b$, it can be shown by substituting $D_{\mathrm{c}} \tan \theta$ and $D^{\prime} \tan \theta$ for $S_{\mathrm{c}}$ and $S^{\prime}$, respectively, in Equation 6 that

$$
S_{\mathrm{R}} / D_{\mathrm{R}}=S / D=\tan \theta .
$$

Equation 1 might be called the primary invariance function, Equation 5 the secondary invariance function, and Equation 7 the direct-response invariance function. When $a=b$ in the weighting equations, the mixture of primary and secondary processes is the same for the response to distance as it is for the response to size. If $a \neq b$, the two processes are given different weights for the size and distance responses, and Equation 7 will not fit the data obtained (Gogel, 1971).

When direct responses are used to measure perceived size and perceived distance, it is frequently found that a constant of proportionality $(C)$ that is often different from unity must be added to Equation 1 if the equation is to fit the data. In this case,

$$
S^{\prime} / D^{\prime}=C \tan \theta,
$$

where $C$ is a situational or observer constant. Research has shown that the perceived ratio $S^{\prime} / D^{\prime}$ is often greater than the physical ratio $S / D$. That is, $C$ in Equation 8 is often greater than one (Foley, 1965, 1968, 1972; Gogel, 1969b, 1977; Gogel, Loomis, Newman, \& Sharkey, 1985). But the finding that $C \neq 1$ does not always mean that Equation 8, rather than Equation 1, is the appropriate equation for expressing the SDIH. According to the above discussion, the finding that $C$ is not equal to unity is not necessarily inconsistent with Equation 1 as an expression of the SDIH, or with Equations 5 or 7, if it is found that $S_{\mathrm{c}}$ and $D_{\mathrm{c}}$ contributed to the direct responses and the weights $a$ and $b$ are not equal. Data obtained by direct responses to size and distance can be discrepant with respect to Equation 1 because (1) secondary as well as primary processes are available, (2) the secondary process contributes to the responses, and (3) the weights given to the primary and secondary processes are different for the responses to distance $(a)$ and to size $(b)$. It follows that if these conditions are present, the data will not provide a valid test of either the primary invariance function (Equation 1), the secondary invariance function (Equation 5), or the direct-response invariance function (Equation 7).

Off-sized perceptions can occur because $S_{\mathrm{c}}$ is varied (by familiarity or suggestion) without producing a similar variation in $S^{\prime}$. Alternately, off-sized perceptions can be varied by changing $S^{\prime}$ with $S_{\mathrm{c}}$ constant. A possible example of this is found in the moon illusion (Kaufman \& Rock, 1962). If the moon at the horizon is perceived as more distant than the moon at the zenith, its perceived size will be larger at the horizon than at the zenith, consistent with the primary process described by the SDIH. But because the horizon and zenith moons are assumed by the observer to be the same size ( $S_{\mathrm{c}}$ is constant), the horizon moon will be seen as a large off-sized object relative to the zenith moon. Thus, consistent with Equation 2, the horizon moon may be reported $\left(D_{c}\right)$ as being less distant than the zenith moon, despite the fact that it is actually perceived as more distant. This phenomenon is also found in the size-distance paradox (Biersdorf, 1966; Epstein et al., 1961; Gruber, 1954; Heineman, Tulving, \& 
Nachmias, 1959; Komoda \& Ono, 1974; Ono, Muter, \& Mitson, 1974), which is illustrated by the following example. A luminous disk is presented in an otherwise dark visual field; shortly after its removal, another disk of a different size (in order to maintain the same visual angle) is presented at a different oculomotor distance. Verbal reports are obtained of the size and distance of the second presentation, relative to the first. Sometimes the result is that, although the reported size of the second presentation is smaller or larger than the first, consistent with the change in the oculomotor cues of distance, the reported distance of the second disk does not vary in the manner expected from the SDIH. Instead, displacing the disk closer or farther from the observer sometimes results in reports of displacement in the opposite direction. In both of these examples, the $S^{\prime}$ of the first object (the zenith moon or the first presentation of the disk) provides the $S_{\mathrm{c}}$ for the second object (the horizon moon or the second presentation of the disk). The second object, because it appears at a different $D^{\prime}$, is perceived to have a size $\left(S^{\prime}\right)$ different from this $S_{\mathrm{c}}$. As specified by Equation $2, D_{\mathrm{c}}$ becomes available to determine the response to distance. In agreement with the previous discussion (Equation 6), in order for the size-distance paradox to occur, the weight (b) given to $S^{\prime}$ relative to $S_{\mathrm{c}}$ must differ from the weight (a) given to $D^{\prime}$ relative to $D_{\mathrm{c}}$. In other words, the observer predominantly uses the perceived size $S^{\prime}$ to determine the response to size, but also predominantly uses the cognitive distance $D_{\mathrm{c}}$ to determine the response to distance. The result, therefore, is interpreted (incorrectly) to be inconsistent with the SDIH. Note, however, that there is an unresolved theory problem here. In the case of successive presentation of stimuli at different values of $D^{\prime}$, it must be determined which $D^{\prime}$ (that from the first or that from the second presentation) is appropriate for Equation 2. The $D_{\mathrm{c}}$ of the horizon moon will be the same as or less than the $D^{\prime}$ of the zenith moon, depending upon whether the $D^{\prime}$ of the horizon moon or of the zenith moon, respectively, is used in Equation 2 . The available data from research on the moon illusion and the size-distance paradox suggest that the first presentation (e.g., the zenith moon) supplies the $D^{\prime}$ as well as the $S_{\mathrm{c}}$ for use in Equation 2.

Clearly, the theory of off-sized perceptions is likely to apply to a wide variety of visual phenomena depending upon the experimenter's ability to distinguish between the primary and secondary processes. The evidence and procedures relevant to this distinction are the concern of the next portion of this paper.

\section{EVIDENCE FOR THE THEORY OF OFF-SIZED PERCEPTION}

\section{Perceived Size With Cue Reduction}

There is evidence that instructions to report the apparent size of familiar objects presented under conditions that are reduced, except for the familiar size (monocular observation in an otherwise dark visual field), will result in reports that often do not approximate the familiar sizes of the objects. In these studies, color transparencies of familiar objects, all located at a constant distance, were used to simulate various distances from the observer. The simulated distance is the distance at which a normal-sized object of that particular kind would have to be placed to produce the visual angle of the transparency. If all information as to the physical distance of the object is eliminated except that of familiar size, the transparency will produce a stimulus identical to that of a normal-sized object physically located at the simulated distance. The advantage of using transparencies at a constant distance, but simulating different distances, is that any differences in the perceived distance obtained from the transparencies must be attributed to the familiar-size cue to distance and not to differences in residual oculomotor cues. The disadvantage of using transparencies at a constant physical distance is that in order for the familiar size to be successful as a cue to distance, it must counter whatever residual oculomotor cues are present to indicate constant physical distance. In the three studies described below, a positive lens that placed the objects accommodatively at optical infinity was used in an effort to further minimize any residual oculomotor cues. If, under these conditions, familiar size is unable to specify perceived size (and thus to determine perceived distance), it is indeed marginal as a cue to perceived size or perceived distance.

In one study simulating distances of 54 to $2,425 \mathrm{~cm}$ (Gogel \& Newton, 1969), observers judged the ratio of the remembered size of the familiar object to its size as perceived in the experiment. In a second study simulating the same distances (Gogel, 1969a), observers reported verbally the perceived sizes and the perceived distances of the objects. The results were as follows. First, in both studies off-sized perceptions occurred frequently. The objects often were judged to have a size that differed from their normal (familiar) size. Second, they were judged as small off-sized objects (they appeared smaller than normal) when their simulated distances were beyond several meters from the observers and as large off-sized objects when the simulated distances were less than several meters. This result is consistent with the interpretation that perceived distance was determined to a very large degree by the SDT, which also determined perceived size in agreement with the SDIH. The reports of distance obtained in the experimental conditions of the second study were corrected by an average calibration curve of verbal reports obtained in a full cue situation (see Mershon, Kennedy, \& Falacara, 1977). The purpose of this calibration was to remove errors unrelated to perceived distance that are characteristically found in verbal reports of distance. The calibrated reports of distance varied with simulated distance, but the range of reported distances was less than that of the simulated distances (also see Gogel, 1974). This pattern of results is consistent with the distance reports being strongly influenced by $D_{c}$, which occurs from the off-sized perceptions (Figure 1). It can be concluded that familiar size, rather than having a pronounced 
effect upon $S^{\prime}$ and $D^{\prime}$ (the primary process), resulted mostly in modifications of $S_{\mathrm{c}}$ and $D_{\mathrm{c}}$ (the secondary process).

Similar results had been obtained earlier in a study using five transparencies of different familiar objects presented in pairs in a frontoparallel plane under otherwise reduced conditions of observation (Gogel \& Mertens, 1968). The perceived distance of each object and the perceived depth between the objects in a pair were measured by verbal reports. The perceived size (width) of each object was measured by the tactile adjustment of the lateral separation of two unseen rods located at the observation position. As in Gogel's (1969a) study, it was found that the reported distance increased with the simulated distance of the objects, again at a reduced rate. At simulated distances greater or less than several meters, the objects tended to appear smaller or larger, respectively, than normal. The results of this study, like those of the two studies discussed above, also are consistent with the idea that the SDT is the main determiner of perceived distance and that the verbal reports of distance are primarily determined by $D_{c}$, in agreement with Equation 2 . These three studies provide clear evidence for the need to distinguish between primary and secondary processes. They also suggest that familiar size, if effective at all, is a weak determiner of perceived size and perceived distance, and has its major effect on the reports of distance produced by $D_{c}$ which operates as a nonperceptual (cognitive) factor.

\section{MEASURING PERCEIVED DISTANCE}

\section{The Head Motion Procedure}

The formal similarity of Equations 1 and 5 suggests the need for procedures to distinguish between primary and secondary processes. One method has been to compare the data obtained from direct and indirect measures of apparent distance, using situations in which off-sized perceptions are likely to be available. Direct measures of perceived distance include verbal reports of distance (Gogel $\&$ Mertens, 1967), pointing with an unseen hand to the apparent distance of the target (Foley, 1975, 1977; Foley \& Held, 1972; Gogel, 1982), relating a comparison distance to the distance of the target (Hastorf, 1950; Ittelson, 1951a, 1951b), pulling a rope through the fingers (Epstein, 1965; Komoda \& Ono, 1974), and throwing darts (Gogel, Hartman, \& Harker, 1957). With direct measures, the relevance of off-sized judgments and thus of $D_{\mathrm{c}}$ to the direct judgment of distance is obvious to the observer, without the observer's recognizing that a cognitive $D_{\mathrm{c}}$ is inappropriate for judging perceived distance. Therefore, as suggested by the studies of Gogel (1969a) and Gogel and Mertens (1968), the perception of the objects as off-sized is apt to influence the direct response to distance, even though the instructions are to indicate perceived distance. Indirect measures are measures in which, from the viewpoint of the observer, the required task does not obviously involve the perceptual dimension (in this case, perceived distance) that is of interest to the experimenter. An established interrelation between perceptions is used to measure one perception (e.g., the perception of distance) by another, different perception (the related perception directly involved in the observer's task). This procedure avoids any tendency on the part of the observer to modify the response to the related perception in order to provide distance measures that are physically rather than perceptually accurate. By using indirect procedures, the experimenter can avoid a cognitive effect, resulting from off-sized perceptions, on measures of perceived distance. An indirect method that has been used to obtain measures responsive to perceived distance unmodified by cognitive distance is called the head motion procedure and is illustrated in Figure 2.

In Figure 2, the prime notations and open circles indicate perceived characteristics of the stimulus object; the notations without primes and the filled circles indicate physical characteristics. The situation illustrated is one in which the observer views the stimulus object while moving the head left and right repetitively through a distance $(K)$. The lines connecting the stimulus object and the observer are construction lines to indicate the direction of the object relative to the observer at the extremes of the lateral head motion. The intersection of these lines specifies the position in space around which the direction between the head and the object pivots as the head moves laterally. The distance of this pivot from the observer is called the pivot distance $\left(D_{\mathrm{p}}\right)$ and $\phi$ is the pivot angle. Figure $2 \mathrm{~A}$ illustrates the situation in which a physically stationary object at a distance $\left(D_{\mathrm{m}}\right)$ appears at a closer distance $\left(D_{\mathbf{n}}^{\prime}\right)$, or at a farther distance $\left(D_{\hat{f}}^{\prime}\right)$. As indicated, in the former case the object will appear to move $\left(+W^{\prime}\right)$ in the same direction as that of the head. In the latter case, the object will appear to move $\left(-W^{\prime}\right)$ in a direction opposite to that of the head. In Figure 2B, the object is physically moved $(-W)$ opposite to the direction of the head movement so as to cancel the apparent motion of the object at its near apparent distance $\left(D_{n}^{\prime}\right)$. In Figure $2 C$, the object is physically moved $(+W)$ in the same direction as the head to cancel the apparent motion of the object at its far apparent distance $\left(D_{f}\right)$. This canceling is equivalent to adjusting the pivot distance $\left(D_{\mathrm{p}}\right)$ to be the same as the apparent distance $\left(D^{\prime}\right)$ of the object. As indicated in Figure 2B and 2C, when $D_{\mathrm{p}}=D^{\prime}, W^{\prime}=0$; that is, the physically moving object appears to be stationary as the head is moved laterally. Thus, the apparent distance $\left(D^{\prime}\right)$ of the stimulus object can be measured indirectly by obtaining a measure of the apparent concomitant motion $\left(W^{\prime}\right)$ when the object is physically stationary (Figure $2 \mathrm{~A}$ ), or by physically moving the object concomitantly with the head motion until $W^{\prime}=0$, at which time $D_{\mathrm{p}}=D^{\prime}$ (Figures 2B and 2C).

From the geometry of Figure 2, it follows that

$$
W^{\prime}=K\left(1-D^{\prime} / D_{\mathrm{p}}\right) \text {. }
$$

Equation 9 is called the apparent distance/pivot distance hypothesis (Gogel, 1982). It contains the basic assump- 


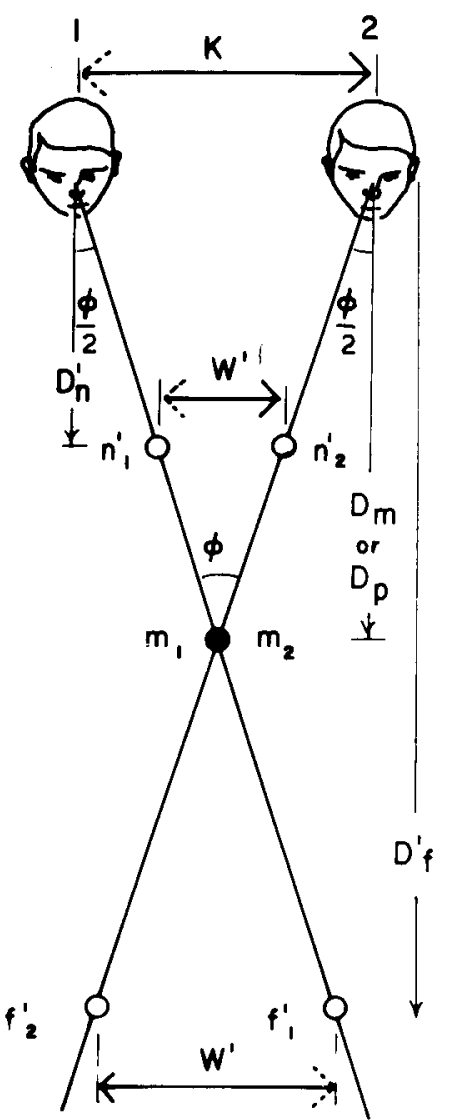

A



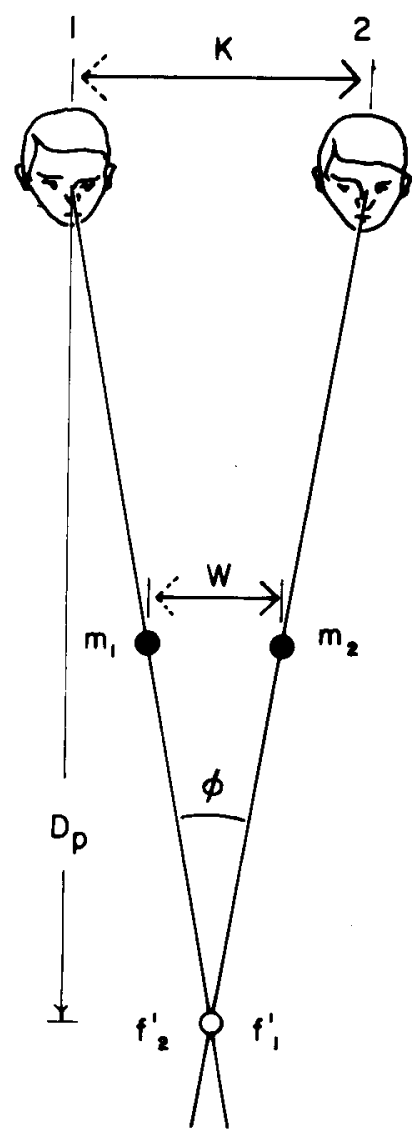

C

Figure 2. Two methods of measuring perceived distance $\left(D^{\prime}\right)$ indirectly, using the head motion procedure. In $A, D^{\prime}$ is measured by determining the apparent motion ( $\left.W^{\prime}\right)$ that occurs concomitantly with the motion of the head as a function of an error in perceived distance, and applying Equation 9. In B and $C, D^{\prime}$ is measured by canceling (nulling out) $W^{\prime}$ by physically moving the object in the direction opposite to that of $W^{\prime}$. (From “Effects of Posthypnotic Suggestion on Perceived Egocentric Distance” by P. J. MacCracken, W. C. Gogel, and G. S. Blum, 1980, Perception, 9, 561-568. Copyright 1980 by Pion Ltd. Adapted by permission.) See text for details.

tion that the perceived change $\left(\phi^{\prime}\right)$ in the direction of the stimulus object per unit of perceived motion $\left(K^{\prime}\right)$ of the head is veridical (i.e., that $\phi=\phi^{\prime}$ and $K=K^{\prime}$ ). This assumption has been tested (Gogel, 1982) and, under the conditions used, it was justified. A more complete form of the apparent distance/pivot distance hypothesis that does not require this assumption but is more cumbersome to apply, is

$$
W^{\prime}=K^{\prime}\left(1-D^{\prime} / D_{\mathrm{p}}^{\prime}\right) \text {, }
$$

where $K^{\prime}$ is the apparent lateral motion of the head and $D_{\mathrm{p}}^{\prime}$ is the apparent distance of the pivot as specified by $K^{\prime}$ and the perceived change in the visual direction $\left(\phi^{\prime}\right)$ (Gogel, 1982). If the above assumptions are met, and if the direction to the object is perceived correctly, it follows from Equation 9 for a physically stationary object $(W=0)$, as in Figure 2A, that

$$
D^{\prime}=D\left(K-W^{\prime}\right) / K,
$$

or, if $W^{\prime}=0$ with $W \neq 0$, as in Figures $2 \mathrm{~B}$ and $2 \mathrm{C}$, it follows that

$$
D^{\prime}=K D /(K-W) \text {. }
$$

Equations 11 and 12 are used in the head motion procedure to calculate $D^{\prime}$ from the observer's judgment of $W^{\prime}$ (with the object stationary) or from the observer's adjustment of $W$ until $W^{\prime}=0$, using the canceling (null) procedure. The applicability, and thus the validity, of the head motion procedure to the detection of changes in perceived distance as expected from changes in distance cues has been shown in a variety of studies (see Gogel, 1981a, 1982; Gogel \& Tietz, 1979; Gogel et al., 1985).

\section{Applications of the Head Motion Procedure}

The head motion procedure for indirectly measuring perceived distance has been applied in several studies involving off-sized perceptions in order to differentiate experimentally between judgments of distance determined by the primary process and the secondary process. These studies will be discussed below. The head motion procedure has also been useful in showing, consistent with the discussion of Figure 1, that the SDT is a primary, not secondary, source of information for perceived distance. 
This was indicated in a study by Gogel and Tietz (1973) in which a point of light was positioned in an otherwise dark visual field at $30,91,183,457$, or $883 \mathrm{~cm}$ from the observer and was viewed with a lateral motion of the head. It was expected that if the concept of the SDT was valid, placing the point at a distance greater or less than the SDT would result in the point's appearing to move with or against the motion of the head, respectively. Only at the physical distance indicated by the SDT would the physically stationary point appear to remain stationary as the head was moved. These expectations are diagrammed schematically in Figure 3, where the solid circles represent physical positions and the open circles perceived positions of the point of light. The SDT was measured by differences between the physical distance $(D)$ and (calibrated) verbal reports $\left(D^{\prime}\right)$, as well as by the interpolated distances at which the obtained frequency of motion with and against the head was equal. Values of the SDT calculated by these methods varied between 1.5 and $3.0 \mathrm{~m}$. Combining the information from this study with the off-sized perceptions obtained by Gogel (1969a) and
Gogel and Newton (1969), it is clear that the SDT can modify both $S^{\prime}$ and $D^{\prime}$ in directions consistent with the SDIH, thus meeting the criterion of a primary process.

Familiar size as a cue of perceived distance. In the three studies of off-sized objects discussed above, which used direct judgments of the apparent size and sometimes the apparent distance of familiar objects (Gogel, 1969a; Gogel \& Mertens, 1968; Gogel \& Newton, 1969), it was found that the report of size often differed from the familiar size, although the reports of distance varied with the simulated distance. It was concluded that to a large extent, the verbal reports of distance must have been determined by cognitive distance $\left(D_{c}\right)$, not perceived distance $\left(D^{\prime}\right)$. If this conclusion is valid, it would have been expected, had the head motion procedure been used, that the measured changes in perceived distance would have been small or absent. This possibility was examined in a study consisting of five experiments (Gogel, 1976). In that study, the perceived distances of familiar objects (transparencies) were measured by the head motion procedure, using the null method, and also by the direct proce-

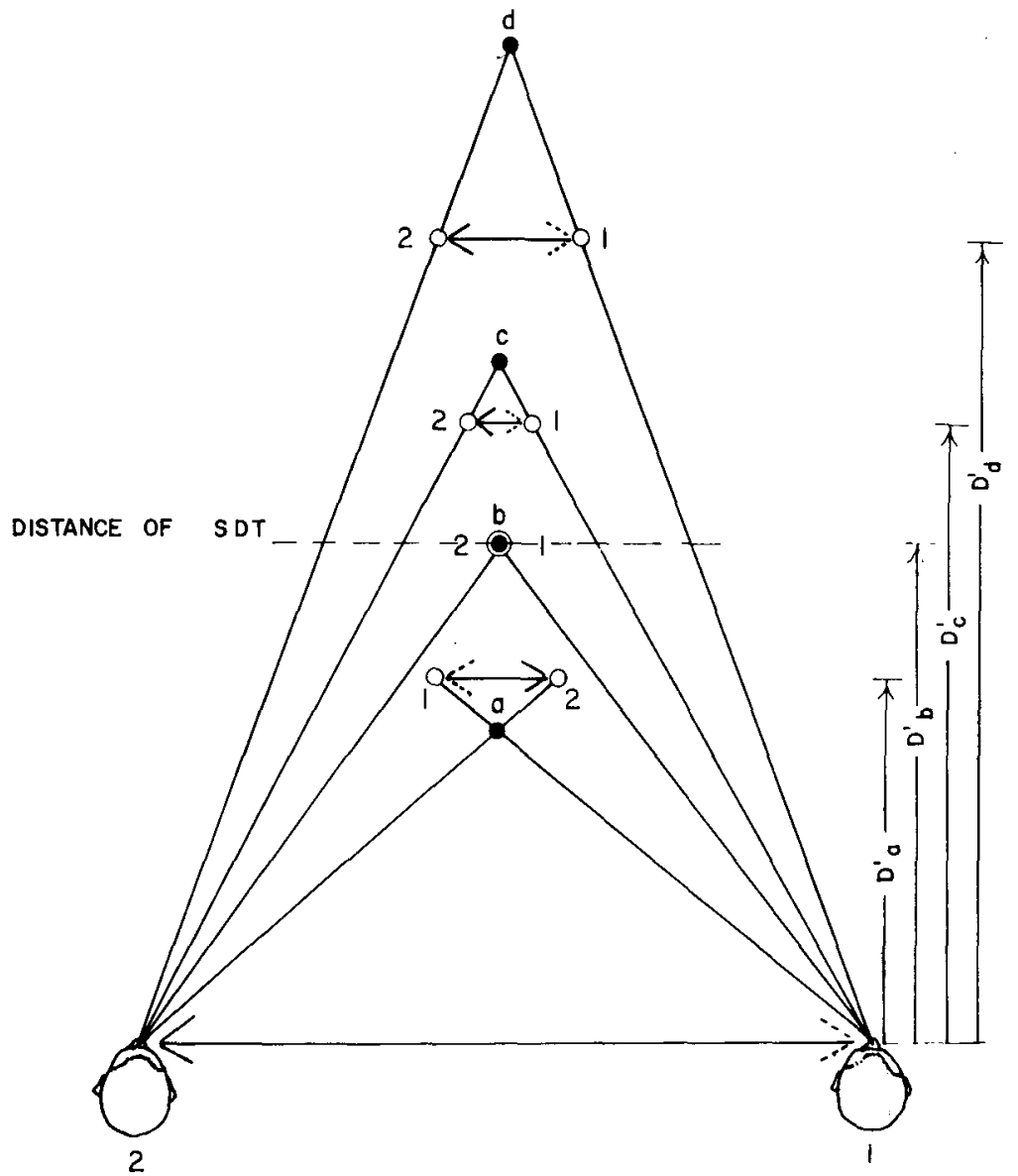

Figure 3. Measuring the distance of the specific distance tendency (SDT) using the head motion procedure. A physically stationary point of light in a dark surround is placed at different physical distances (a through d) from the observer until it no longer appears to move against or with the head as the head is moved laterally. The physical distance of the point at which this occurs is the distance of the SDT. 
dure of verbal reports. The luminous transparencies were presented one at a time at optical infinity, in an otherwise totally dark visual field, and were viewed monocularly. In one experiment, three transparenciesrepresenting a key, sunglasses, and a guitar, and simulating distances of 63,185 , and $1,236 \mathrm{~cm}$, respectively, from the observer-were presented successively. In three additional experiments only the key and guitar were used. In a fifth experiment, two transparencies-representing a playing card at two different simulated distances-were presented successively. The apparatus for the head motion procedure permitted the pivot distance of the familiar object to be varied until the null criterion of no apparent object motion was achieved. In addition, the observers were instructed in the first four experiments, in which the shapes of the objects were irregular, to imagine a short vertical line on each end of the width of the object and to verbally indicate the apparent lateral distance between the lines. In the fifth experiment, the height of the playing card was judged. Estimates also were obtained of the remembered size of normal familiar objects of the same kind as those pictured in the transparencies. The verbal reports of apparent distance in the experimental situations again were calibrated, using distance estimates obtained under full cue conditions (Mershon et al., 1977). It was found from both the head motion procedure and the verbal reports of distance that perceived distance increased as simulated distance increased, indicating that familiar size was at least somewhat effective in modifying perceived distance. However, the geometric mean of the ratio of the perceived distance of the guitar to the key, averaged over the four relevant experiments, was only 1.4 for the head motion procedure but 5.8 for the verbal reports of distance. Both ratios were considerably less than the 19.6 ratio of the simulated distance of the guitar to the key. The geometric mean (again averaged over the four experiments) for the ratio of the remembered familiar size to the measured perceived size $\left(S_{\mathrm{c}} / S^{\prime}\right)$ was .71 for the key and 5.6 for the guitar. The key was perceived as a large off-sized object, whereas the guitar was perceived as a small off-sized object. The off-sized perceptions that occurred were consistent with the modification of perceived distance away from the simulated distance and toward the distance defined by the SDT. The considerably larger ratios of perceived distance obtained from verbal reports, compared with perceived distance as measured by the head motion procedure, indicate that a very substantial portion of the verbal report of distance could be attributed to $D_{\mathrm{c}}$, with the implication that this cognitive effect was produced by the off-sized perceptions.

Experiment 5 (Gogel, 1976) differed from the first four experiments in that the successive presentations represented the same kind of familiar object (a large and a small playing card), simulating distances of 89 and $222 \mathrm{~cm}$ (a ratio of 2.5). This permitted the relative size cue to occur between the successive presentations. This addition of the relative size cue to the familiar size cue to distance was expected to result in larger differences in perceived distance between the second presentations than between the first presentations of the two sizes of cards (Gogel et al., 1957). This expected result was obtained with both the head motion procedure and direct reports of distance. However, also as expected, the ratios of the geometric means for both the first and second presentations were smaller for the head motion procedure (1.2 and 1.7, respectively) than for direct reports of distance (1.5 and 3.6). Also, according to verbal reports of size, the geometric mean of $S_{\mathrm{c}} / S^{\prime}$ was 1.0 for the large card and 0.7 for the small card. Again, this evidence implies that off-sized perceptions and $D_{c}$ contribute to the verbal reports of distance. Also, the differences in the results between the first and second presentations demonstrate the sensitivity of the head motion procedure to the relative size cue to perceived distance.

Gogel and Da Silva (in press) compared the effect of objective and apparent instructions on the reports of size and distance for playing cards and blank rectangles. The study, except for the instructions, is patterned after a study by Fitzpatrick et al. (1982) in which it was found that familiar size was a veridical cue of size and distance, at least for distances near the observer (Fitzpatrick et al. used instructions that essentially were objective rather than apparent). In Gogel and Da Silva's study, each observer was presented with either a physically normal-sized playing card or a blank rectangle of the same physical size as the playing card, at one of three physical distances $(56,107$, or $149 \mathrm{~cm}$ ) under otherwise reduced conditions. The viewing was monocular through a small restrictive aperture. Distance was indicated verbally and size tactually. For both objective and apparent instructions, it was found that reported distance remained essentially constant for the blank rectangle and increased with physical distance for the playing card. For both kinds of instructions, the judged size of the rectangle decreased as the physical distance increased. The judged size of the playing card remained constant for objective instructions, but decreased with increasing physical distance for apparent instructions. This pattern of results can be explained by an egocentric reference distance causing the perceived distance $\left(D^{\prime}\right)$ of both the playing card and the blank rectangle to appear more nearly constant than would be expected from their different physical distances. As a consequence, the perceived size $\left(S^{\prime}\right)$ of the physically more distant objects of both kinds appeared smaller than the perceived size of the physically less distant objects. However, because only the playing card had a familiar size $\left(S_{\mathrm{c}}\right)$, only the playing card appeared to be off-sized. This permitted the verbal report of the distance of the playing card, using $D_{c}$, to closely agree with the card's physical distance. Were it not for the off-sized responses to the playing card obtained from apparent instructions, it could have been concluded (mistakenly) that familiar size was an excellent cue of perceived size and, for this reason, an excellent indicator of perceived distance. It also appears, from this experiment, that although the weight given $S^{\prime}$ as compared with $S_{\mathrm{c}}$ was increased by the apparent instructions, the weight given 
$D^{\prime}$ as compared with $D_{\mathrm{c}}$ was not. It is likely that each of the components of $S_{\mathrm{c}} / S^{\prime}$ is available to awareness, but that $D_{\mathrm{c}}$ and $D^{\prime}$ are not easily discriminated by the observer in forming a direct report of distance.

Taken together, the above studies of off-sized perceptions provide considerable support for the distinction between primary and secondary processes in the direct response to size and distance as characterized by Equations 1 through 6. One of the salient findings is that offsized perceptions resulting from differences in size information from the two processes are often available. This availability increases as cues for veridical perceived distance, and thus veridical perceived size, are reduced. Such reductions can occur not only in many experimental situations and in extended visual fields, but also as a function of local conditions. For example, the spatial position of a chandelier suspended from the ceiling of a room is likely to be less accurately localized perceptually in space than is a chair standing on a patterned floor. Equally important, eye fixation and, to some degree, attention have been shown to produce systematic errors in the perceived distance of the nonfixated or nonattended object as measured by the illusory motions $\left(W^{\prime}\right)$ of these objects concomitant with a lateral motion of the head (Gogel, 1979; Gogel \& Tietz, 1977). It is likely that off-sized perceptions can occur rather frequently, even under multicue conditions.

Suggested size as a determiner of perceived distance. It seems, according to the study by Gogel (1976), that familiar size can have an effect, although quite a limited one, on perceived distance as found by measurements obtained with the head motion procedure. It might be expected that casual suggestion, such as was used in Hastorf's (1950) study, would be even less effective. This was investigated in a study (Gogel, 1981b) using both the head motion procedure and direct verbal reports of perceived distance. A blank target, either circular or rectangular in shape, was viewed monocularly at a constant physical distance $(120 \mathrm{~cm})$ in an otherwise dark visual field. Size suggestions were provided by showing the observer a large or small familiar object of the same shape as the target and informing the observer that the target was the same size as the familiar object. The familiar object was shown to the observer prior to target presentation and was available tactually but not visually during target presentation. A different group of observers was used with each of the four suggested size and target combinations. Two kinds of measures of the perceived distance of the target were obtained. One, a direct measure, consisted of verbal reports of perceived distance. The other, the head motion procedure, used the null criterion in which the pivot distance was adjusted until the observer reported no apparent motion of the target. The results were unequivocal. The verbal reports of distance clearly increased with increases in suggested size. The verbal reports indicated that suggested size modified perceived distance, and, by implication from Equation 1, perceived size. The head motion procedure, on the other hand, clearly indicated that suggestion had no discernible effect on perceived distance, and thus, according to Equation 1, no effect on perceived size. These results can be explained in terms of off-sized perceptions as follows. As shown by the head motion measures of perceived distance, suggestion did not modify the perceived distance $\left(D^{\prime}\right)$ of the target (the blank rectangle or disk). According to the measures of $D^{\prime}$ from the head motion procedure, the target at $120 \mathrm{~cm}$ was perceived on the average to be at a somewhat greater distance, consistent with the effect expected from the SDT. This $D^{\prime}$, together with the visual angle of the target, determined an $S^{\prime}$ that often differed from the suggested size $\left(S_{\mathrm{c}}\right)$. The resulting off-sized judgments $\left(S_{\mathrm{c}} / S^{\prime}\right)$ produced values of $D_{\mathrm{c}}$ that often differed from $D^{\prime}$. Consequently, unlike the results obtained from the head motion procedure, the suggested increase or decrease in the size of the target resulted in a verbally reported increase or decrease in its distance. Direct reports of distance, but not perceived distance (and presumably not perceived size), were modified by the casual suggestions of size provided by the familiar examples.

Off-sized perceptions and optical expansion. It seems from the studies discussed so far that familiar size, or size suggestions from the experimenter communicated by labeling, can modify verbal reports of distance by producing off-sized judgments. In the former case, the modifications of the verbal reports to a very limited degree involve some change in perceived distance. In the latter case, the changes are completely cognitive. In a recent study (Swanston \& Gogel, 1986), it was concluded that off-sized perceptions are involved in the perception of motion in depth from optical expansion or contraction (optical size changes), and, as indicated by measures using the head motion procedure, this depth motion can be entirely perceptual.

If an optical expansion (or contraction) is completely effective in determining apparent motion in depth, it is expected that the perception will be of an object of constant size moving toward (or away from) the observer. This expectation follows from the SDIH, in which, according to Equation 1, the amount of perceived motion in depth $\left(d^{\prime}\right)$ between the perceptually farther $\left(D_{f}^{\prime}\right)$ and the perceptually nearer $\left(D_{\mathrm{n}}^{\prime}\right)$ distance of the stimulus is

$$
d^{\prime}=D_{\mathrm{i}}^{\prime}-D_{\mathrm{n}}^{\prime}=S_{\mathrm{f}}^{\prime} / \tan \theta_{\mathrm{f}}-S_{\mathrm{n}}^{\prime} / \tan \theta_{\mathrm{n}},
$$

where $S_{\mathrm{f}}^{\prime}$ and $S_{\mathrm{n}}^{\prime}$ are the perceived sizes of the stimulus at the far and near perceived distances, and $\theta_{\mathrm{f}}$ and $\theta_{\mathrm{n}}$ are the visual angles of the stimulus at these distances. According to Equation 13, a tradeoff must occur between the change in perceived size and the change in perceived distance. In particular, if $S_{\mathrm{n}}^{\prime} / S_{\mathrm{f}}^{\prime}=\tan \theta_{\mathrm{n}} / \tan \theta_{\mathrm{f}}$ (or, to a close approximation for visual angles that are not too large, if $S_{\mathrm{n}}^{\prime} / S_{\mathrm{f}}^{\prime}=\theta_{\mathrm{n}} / \theta_{\mathrm{f}}$ ), it follows that $d^{\prime}=0$. The equation for specifying from secondary processes the simu- 
lated motion in depth with optical expansion is as follows (as found by applying Equation 2 and simplifying using Equation 1):

$$
\begin{aligned}
d_{\mathrm{c}} & =D_{\mathrm{c}}(\text { far })-D_{\mathrm{c}}(\text { near }) \\
& =S_{\mathrm{c}}\left(\tan \theta_{\mathbf{n}}-\tan \theta_{\mathrm{f}}\right) / \tan \theta_{\mathbf{n}} \tan \theta_{\mathrm{f}},
\end{aligned}
$$

where $S_{\mathrm{c}}$, the perceived size at the beginning of the expansion, acts as a standard for subsequent off-sized perceptions occurring throughout the optical change. This constancy of $S_{\mathrm{c}}$ throughout an optical change in a given direction, however, does not require a long-term memory of the perception of size at the beginning of the change. Instead, each successive instant of optical change provides the standard for the next instant, resulting in a process equivalent in its effect to carrying the original memory of the starting $S_{\mathrm{c}}$ forward in time. In Swanston and Gogel's (1986) study, equations derived from Equation 14 were compared with Equation 13 in their ability to predict the data obtained from the optical changes. Equation 14 does not imply a tradeoff between $d_{\mathrm{c}}$ and changes in perceived size. Instead, the greater the perceived changes in size for a given $S_{\mathrm{c}}$, the greater will be the reported motion in depth attributable to the secondary process.

Swanston and Gogel's (1986) study involved four experiments. The optical size changes were presented on a monitor at a constant distance from the observer, in an otherwise dark visual field. In Experiment 1, using monocular observation, the observers indicated the amount of perceived motion in depth by tactile adjustment of the sagittal separation of two posts. The perceived size at the simulated near and far distances was indicated by tactile adjustment of the lateral separation of two posts. The results indicated a substantial motion in apparent depth, but also a ratio of perceived sizes equal to or greater than the ratio of retinal sizes. This result is contrary to Equations 1 and 13.

The conditions of Swanston and Gogel's (1986) Experiment 2 generally were similar to those of Experiment 1, except that the observation was binocular and convergence and optical expansion, when both were present, were in agreement or were opposed in their effect on apparent motion in depth. Also, in Experiment 2, the head motion $\left(W^{\prime}\right)$ procedure was used in addition to the sagittal post adjustments, to measure changes in perceived distance. Lateral adjustments of the post by touch again provided measures of perceived size. The results relevant to the present discussion were as follows: (1) The ratios of perceived size were similar to the ratios of retinal size. (2) Substantial amounts of depth motion were reported by the direct method of sagittal adjustment of the posts. (3) The indirect head motion procedure provided some evidence that the depth motion reported by the direct method of sagittal adjustment of the posts involved perceived depth. This evidence was most convincing in that the effect of convergence changes, which clearly were perceptual, on apparent depth motion was canceled by the changes in optical size when measured by the head motion rather than by the direct sagittal adjustment. This result also suggests that when depth motion from optical expansion is in opposition to another cue (e.g., convergence), the seeming dominance of optical expansion as measured by a direct procedure can be cognitive rather than perceptual. This experiment, like Experiment 1, indicated that the SDIH as expressed by Equation 1 cannot predict the responses from optical expansion. In addition, Experiment 2 offered some evidence, provided by the head motion procedure, that this failure of the SDIH cannot be explained by asserting that the perceived depth was cognitive rather than perceptual and that, therefore, it was not relevant to the SDIH, which is concerned with perceptions only.

Swanston and Gogel's (1986) Experiment 3 simulated motions in depth from optical expansion and contraction in the absence of other cues to perceived motion in depth. The expanding or contracting stimulus (the optical size change) was at a constant physical distance in an otherwise dark visual field and was viewed monocularly. Direct (sagittal) measures of perceived depth and indirect head motion measures were obtained, again, by computing $D_{f}^{\prime}-D_{n}^{\prime}$ from values of $W^{\prime}$. Lateral post adjustments were used to measure perceived size at the near and far simulated distances. The main results were (1) that the ratio of perceived sizes was again similar to the ratio of the terminal visual angles of the stimuli, and (2) that substantial perceived motions in depth occurred, as measured by the indirect (head motion procedure) as well as the direct (sagittal adjustment) method. The magnitudes of the results from the direct and indirect procedures were very similar. These results are inconsistent with the tradeoff between perceived size and perceived distance implicit in the SDIH as expressed by Equation 13. They also provide strong support for the possibility that offsized perceptions, under the proper conditions, can have a pronounced effect on perceived, in contrast to cognitive, depth.

The data from all three experiments (Swanston \& Gogel, 1986) can be explained in terms of the interrelation of primary and secondary processes as expressed in Equations 1, 2, and 14. In most of the situations used, distance cues other than the optical size changes were constant. These, together with the STD, determined an egocentric reference distance. This constant perceived distance $\left(D^{\prime}\right)$ resulted in a changing perceived size $\left(S^{\prime}\right)$ proportional to the change in visual angle, as is indicated by the finding that $S_{\mathrm{n}}^{\prime} / S_{\mathrm{f}}^{\prime}$ was equal approximately to $\theta_{\mathrm{n}} / \theta_{\mathrm{f}}$. This $S^{\prime}$ and $D^{\prime}$ is the contribution of the primary process. At the start of an optical expansion, for example, the $S^{\prime}$ resulting from the primary process provides the $S_{\mathrm{c}}$ for the subsequent expansions. Thus, following the starting presentation, the line stimulus that is increasing in perceived size $\left(S^{\prime}\right)$ is judged to be an increasingly large offsized object (decreasing $S_{\mathrm{c}} / S^{\prime}$ ). This perception of an increasingly large off-sized object provides decreasing values of $D_{c}$ (according to Equation 2), which results in the response that the stimulus is moving in depth toward the observer as the optical expansion continues. It is of 
special interest that the motion in depth, particularly in Experiment 3, was perceptual, as is indicated by the measurement of this motion using the head motion procedure. In Experiment 4 of the study, it was predicted and found, consistent with equations derived from Equation 2, that increasing the starting perceived distance $\left(D^{\prime}\right)$ increased the magnitude of the perceived depth motion. Throughout the study, the direction of the effects predicted from Equation 2 and from equations derived from Equation 2 provided support for the contribution of secondary processes (as specified by the theory of off-sized perceptions) to the perceptions associated with optical changes in stimulus size. Multilevel theories of processing size and distance information, in addition to the model summarized here, have been proposed in order to explain seeming failures of the SDIH (see Higashiyama, 1977, 1979, 1983; Komoda, 1970; Komoda \& Ono, 1974; Ono et al., 1974). However, none of these theories distinguish between perceptual and cognitive factors or consider the role of offsized judgments in direct responses to distance. Therefore, all are unlikely to explain the results obtained from the studies of familiar size and casual suggestion using the head motion procedure (Gogel, 1976, 1981b) or the results from optical expansion obtained in Swanston and Gogel's study (1986).

\section{THE IMPORTANCE OF PERCEIVED DIRECTION}

\section{A Modification of Equation 1}

Studies in which a distance response is determined either partially or completely by off-sized perceptions provide no challenge to the SDIH as long as the modification is nonperceptual as measured by the head motion procedure. The results from the optical expansion study (Swanston \& Gogel, 1986), however, have serious implications for the SDIH. In that study, two sources of information regarding distance were distinguished. Both were identified as producing perceptions of distance as measured by the head motion procedure. One of these involved the relation between perceived size and perceived distance present at the start of the optical change. This primary process was consistent with the SDIH. The other, the response of motion in depth, was not accompanied by the perceived changes (or lack of changes) in size expected from the SDIH. These two sources of perceived distance were in conflict. It is concluded from the data that the constant oculomotor cues associated with the primary process tended to restrict the perceived motion in depth resulting from the optical change. The constant perceived depth expected from the oculomotor cues was in opposition to the perceived motion in depth from the secondary process. Conflicts between different sources of distance information are not unusual and can be resolved by the visual system. For example, discrepant depth information from perspective and binocular disparity cues can result in a perception to which both contribute (Gillam, 1968). Thus, a resolution by means of a weighting process of the conflict between the discrepant perceived distances from primary and secondary sources is to be expected. What is puzzling is why the SDIH, which is so useful in predicting a variety of spatial phenomena, is unable to predict the results obtained with optical expansion even though the distance changes are indeed perceptual, as demonstrated by the head motion procedure. There may be a solution to this problem. It may be that Equation 1 or Equation 8, which usually are considered to represent the SDIH, needs to be modified. It has been suggested by McCready $(1965,1985,1986)$ that the term perceived visual angle $\theta^{\prime}$ (a change in perceived direction) be substituted for the term physical visual angle $\theta$ in the specification of the SDIH. In that case, the equation for the SDIH becomes

$$
S^{\prime} / D^{\prime}=\tan \theta^{\prime} .
$$

In the following discussion we consider the rationale for the substitution of Equation 15 for Equation 1. We conclude that there are reasons for considering the perception of changes in direction to be an integral part of the SDIH. It will be noted that Equation 15 offers the possibility of reconciling the SDIH with the results obtained in the study by Swanston and Gogel (1986). This possibility needs to be tested. We also suggest, however, that some of the phenomena used by McCready to support the need for Equation 15 can be explained by the two-process theory described in this paper.

\section{Perceived Direction and the SDIH}

It will be recalled that two of the factors in the head motion procedure, as indicated by Equation 10, are the observer's perception of the change in the direction of the target stimulus relative to the position of the head $\phi^{\prime}$ and the observer's sensing of the magnitude of head motion. To simplify the application of Equation 10, it was assumed (in Equation 9) that these two perceptions are accurate throughout the head motion, for example, that $\phi$ and $K$ in Figure 2 are equal to $\phi^{\prime}$ and $K^{\prime}$ (i.e., effectively, $D_{\mathrm{p}}=D_{\mathrm{p}}^{\prime}$ ). Both assumptions have been tested and found to be valid under the experimental conditions to which the head motion procedure usually has been applied (Gogel, 1982), permitting the more convenient formulation of the head motion procedure (Equation 9) to be used. It can be shown that the SDIH is a special case of the apparent distance/pivot distance hypothesis as expressed by Equation 9 or Equation 10, and, as such, it involves the same assumption regarding the perception of changes in direction. Consider the case in which the observer views a target that is physically moving laterally $(W)$ concomitant with the lateral motion of the head and is located at the physical distance $D$. It can be shown from Equation 9 (see Gogel, 1982) that

$$
W^{\prime}=\left(K D-K D^{\prime}+W D^{\prime}\right) / D .
$$

If the head is stationary, $K=0$, and it follows from Equation 16 that

$$
W^{\prime} / D^{\prime}=W / D
$$


Equation 17 is the same as Equation 1 except that $W$ and $W^{\prime}$ refer to the physical and perceived lateral extent of motion of the stimulus object, whereas $S$ and $S^{\prime}$ refer to the physical and perceived lateral extent of size of the stimulus object. The validity of equating extents of motion and extents of size in the SDIH has been shown experimentally by Rock, Hill, and Fineman (1968) and by Wist, Diener, and Dichgans (1976). Since Equations 9 and 16 apply only if $K^{\prime}=K$ and $\phi^{\prime}=\phi$, it follows that Equations 1 and 17, as special cases of Equations 9 and 16 , apply only if $K^{\prime}=0$ and $\theta^{\prime}=\theta$. If the observer does not perceive the change in direction to the ends of the stimulus correctly (i.e., if $\theta^{\prime} \neq \theta$ ), Equation 15 is required to represent the SDIH. It seems that Equation 15 is the most general, and therefore possibly the most valid, expression of the SDIH, just as Equation 10 is the most valid expression of the apparent distance/pivot distance hypothesis.

A geometrical illustration of the relation between the head motion procedure and the SDIH is shown in Figure 4. Figure 4A is similar to Figure 2A except that all the terms are perceptual to represent Equation 10. The situation illustrated is one in which the observer senses (perceives) his head to move laterally through a distance, $K^{\prime}$ (very likely because his head physically moves through

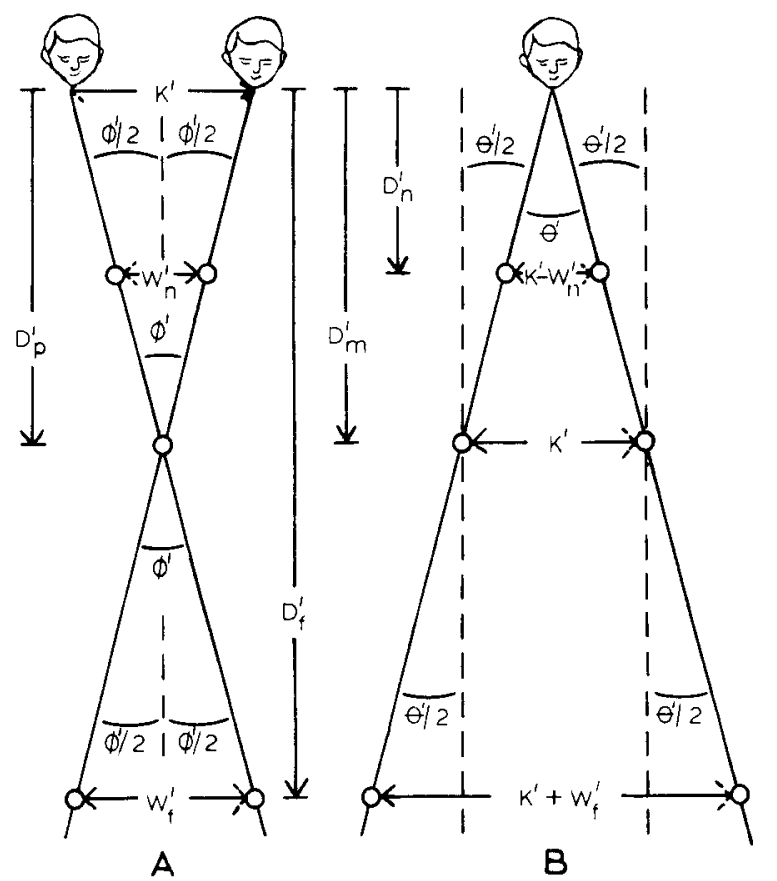

Figure 4. Illustration of the commonality of visual requirements in (A) a situation applying the apparent-distance/pivot-distance hypothesis (Equation 10) and in (B) a situation applying the SDIH (Equation 15). If the perceived distances $\left(D_{\mathrm{n}}^{\prime}, D_{\mathrm{m}}^{\prime}\right.$, and $\left.D_{i}\right)$ are the same in situations $A$ and $B$, Equations 10 and 15 can be combined. In this case, the perceived lateral motion (illustrated in B) of the point located at each of the apparent distances equals $\left(K^{\prime} \pm W^{\prime}\right)\left(\tan \theta^{\prime} / \tan \phi^{\prime}\right)$. If $\theta^{\prime}=\phi^{\prime}$, as shown in the figure, the perceived extent of motion equals $K^{\prime} \pm W^{\prime}$, as is illustrated also in $B$. a distance, $K$ ), while viewing a target that appears to change in its direction from the observer through the angle $\phi^{\prime}$ (very likely because the object is physically stationary). In this case, if the object appears at a near distance $\left(D_{n}^{\prime}\right)$ it will appear to move $\left(W^{\prime}\right)$ in the same direction as the sensed motion of the head. If it appears at $D_{\mathrm{m}}^{\prime}$, it will appear to remain stationary, and if it appears at $D_{f}^{\prime}$, it will appear to move against the sensed motion of the head. Figure 4B illustrates the phenomenal conditions representative of the SDIH as expressed by Equation 15. In this case the head is sensed to be stationary (very likely because it is physically stationary) and the object appears to move laterally (very likely because it is physically moved) through an apparent extent, $K^{\prime}$, with $K^{\prime}$ the same in the two situations represented by $A$ and $\mathrm{B}$. If $D_{\mathrm{n}}^{\prime}, D_{\mathrm{m}}^{\prime}$, and $D_{\mathrm{f}}^{\prime}$ also are the same in Figures $4 A$ and $4 B$, then $\theta^{\prime}=\phi^{\prime}$ and the difference in the perceived motions of the object between $D_{\mathrm{f}}^{\prime}$ and $D_{\mathrm{n}}^{\prime}$ is the same in the two situations. Clearly, the same abilities to perceive direction and motion or lateral extent are required for the SDIH and the head motion procedure and, theoretically, the perceptual results obtained from one should be highly related to those from the other. As indicated, under most circumstances, perceived and physical direction are in correspondence. But Equations 10 and 15 are more general than Equations 9 and 1 in that they are expected to apply whether this correspondence is present or not.

As suggested by Figure 4, it is expected that the measures of $D^{\prime}$ computed from Equations 9, 1, and 17 will be similar or at least highly correlated. In one study (Gogel et al., 1985), the observers adjusted a frontoparallel extent to a constant subjective size, and from these adjustments measures of $D^{\prime}$ were obtained, using the SDIH. Measures of $D^{\prime}$ were also obtained from $W^{\prime}$ in the same situation, using the head motion procedure and Equation 9. Although the measures of $D^{\prime}$ from the SDIH were consistently larger than those obtained from the head motion procedure, the average data from the two procedures were very highly correlated $(r=.981)$. In another study (Gogel \& Tietz, 1979), the perceived distance of a point stimulus in a dark surround was varied by changing the physical distance of the point. The point was viewed monocularly or binocularly, using horizontal head motion, under two conditions. In one condition, the point was stationary or moved only horizontally $(W)$, and perceived distance $\left(D^{\prime}\right)$ was computed from measurements of $W^{\prime}$, using Equation 9 . In the second condition, a vertical component of motion was added that differed at the different physical distances so that it always produced the same angle of vertical motion on the eye of the observer. The horizontal and vertical components of concomitant motion resulted in the point's physically moving at a slant, with the apparent slant modified by the apparent distance of the point. The resulting apparent slant was the vector sum of the apparent horizontal component $\left(W^{\prime \prime}\right)$ predictable from Equation 9 and the apparent vertical component $\left(S^{\prime}\right)$ predictable from Equation 1 . The perceived distance $\left(D^{\prime}\right)$ in the second condition was computed from 
measurements of perceived slant and therefore depended upon the validity of both the SDIH and the head motion procedure. Only if the SDIH provided measures of $D^{\prime}$ similar to those obtained from the head motion procedure could the measured values of $D^{\prime}$ from the two conditions agree. The average results obtained were similar (see Gogel \& Tietz, 1979, Figure 3) and highly correlated, and provide support for the similarity of the processes underlying the apparent distance/pivot distance hypothesis and the SDIH. It seems, therefore, that the perception of changes in direction is an important variable in the SDIH, as it is in the head motion procedure, and that Equation 15 is more likely than Equation 1 to provide a valid expression of the SDIH.

\section{Possible Added Reasons for Using $\theta^{\prime}$ in the SDIH}

McCready $(1965,1985,1986)$ advocated the use of $\theta^{\prime}$ rather than $\theta$ (or, in McCready's terms, $v$ rather than $V$ ) in the specification of the SDIH in order to remove the inconsistencies obtained with Equation 1. Certain phenomena involving responses to size and distance, because they are inconsistent with the SDIH as expressed by Equation 1, are classified as paradoxes. One of these is concerned with changes in the judgment of size and distance associated with changes in oculomotor cues to the distance of the target. For a target of constant visual angle, a decrease or increase in the reported size of the target as a consequence of oculomotor changes to a nearer or farther distance is consistent with the results expected from the SDIH; a decrease in reported size is called AC micropsia, and an increase is called AC macropsia. As noted previously, however, it is sometimes found that the reported distance of the target is inversely related to its reported size. The result (the size-distance paradox) is not in agreement with Equation 1. A similar kind of dissociation of the relation expressed by Equation 1 between judged size and distance is found with geometrical illusions of extent, such as the Müeller-Lyer and Ebbinghaus illusions. In these illusions, frontoparallel extents of equal visual angle are perceived as unequal in size but not necessarily as located at different perceived distances from the observer, as required by Equation 1. McCready suggests that the paradoxes associated with oculomotor changes and these geometrical illusions would be avoided if perceived visual angle were substituted for physical visual angle in the SDIH. However, as is clear from the previous discussion, a difference between $\theta^{\prime}$ and $\theta$ is not the cause of a number of seeming failures of Equation 1. As noted, Equation 1 is expected to fail if the cognitive factors $S_{\mathrm{c}}$ and $D_{\mathrm{c}}$ resulting from off-sized perceptions influence the reports of distance with different values of $a$ and $b$ used in the weighing functions (Equations 4 and 5 ). It is necessary to use procedures (such as the head motion procedure and apparent instructions) that minimize cognitive effects. If any data discrepancies remain, it is possible that these can be resolved by substituting measured values of $\theta^{\prime}$ for $\theta$ in Equation 1 . This possibility would have to be demonstrated by actual measures of $\theta^{\prime}$ obtained in these cases and in the cases of the geometrical illusions. It seems that this has not yet been done. If $\theta^{\prime}$ and $\theta$ are indeed found to differ by actual measurement, it is reasonable to expect from geometrical considerations, as shown in Figure 4, that Equation 15 would be more compatible with the results than would Equation 1.

From his discussion of illusions not produced by differences between $\theta^{\prime}$ and $\theta$, it seems that McCready expected assumed size to modify perceived distance (McCready, 1965, Theorem 4, and 1985, p. 327) and assumed distance to modify perceived size (McCready, 1965, Theorem 5). The present article presents clear evidence against the former expectation in the case of casual suggestion (Gogel, 1981b), and evidence for only a small effect of assumed size in the case of familiar size (Gogel, 1976). Some evidence against the notion that assumed distance can modify perceived size is found in a study by MacCracken, Gogel, and Blum (1980). In that study, verbal reports of the distance of a point of light observed binocularly in a dark surround were modified by posthypnotic suggestions, but no modification of perceived distance, as measured by the head motion procedure, was evident. If suggestions provided under hypnotic conditions are unable to modify perceived distance, it seems unlikely that less stringent methods of introducing suggestions regarding distance would modify perceived size.

It will be noted that Equation 15 does not contain a constant of proportionality $(C)$ as does Equation 8 . The fact that $C$ in Equation 8 is often greater than unity need not prevent Equation 8 from representing the SDIH. The angle $\theta$ is essentially proportional to proximal size, and the proximal size is readily conceived to undergo transformations prior to its contribution to the perception of the ratio of perceived size to perceived distance. The case of Equation 15, however, is somewhat different in that $\theta^{\prime}$ is available at the level of perception. It might be expected that $S^{\prime} / D^{\prime}$ should not be merely proportional to $\tan \theta^{\prime}$ but should equal $\tan \theta^{\prime}$. But there is evidence to question this. In a study by Foley (1968) in which only oculomotor cues were available to determine perceived distance, the observers adjusted the perceived size of a frontal extent to be half as long as its distance from themselves. The average result was that $S^{\prime} / D^{\prime}$ was more than twice as large as $\tan \theta$. In a previous study (Foley, 1965), under the same conditions, observers estimated depth angles in degrees, adjusted the angles to a given value in degrees, and adjusted one angle to be half the value of another. All of these methods gave results that indicated that $\theta^{\prime}$ is only slightly (about $10 \%$ ) greater than $\theta$. If $S^{\prime} / D^{\prime}$ is considerably larger than $\tan \theta$, whereas $\theta^{\prime}$ is about equal to $\theta$, it follows that $S^{\prime} / D^{\prime}$ also is larger than $\tan \theta^{\prime}$. In this case, Equation 15 should contain a value of $C>1.00$.

\section{Application of Equation 15 to Optical Change}

In the study by Swanston and Gogel (1986), Equation 1 failed to predict the perceived motion in depth from opti- 
cal expansion even though the head motion procedure affirmed that the depth motion was perceptual. Perhaps the reason for the failure of Equation 1 was that off-sized perceptions associated with the continuously changing stimulus caused $\theta^{\prime}$ to differ substantially from $\theta$. This would be consistent with Equation 15 if it were found that $\theta^{\prime}$ increased or decreased at a faster rate than the physical angular change. In particular, in the case of optical expansion, the apparent visual angle would need to increase more rapidly than the physical visual angle as the expansion proceeded. In the case of optical contraction, the apparent visual angle would need to decrease more rapidly than the physical visual angle as the contraction proceeded. Whether or not such changes occurred in Swanston and Gogel's study is not known. This possibility could be tested by comparing perceived visual angle at both the near and far simulated distances of the optical change prior to and immediately following an optical change. A study of this kind could not only reassess the failure of the SDIH to apply to optical expansion or contraction, but could also provide a test of the value of applying Equation 15 to situations in which Equation 1 fails. Such a study is being prepared.

\section{SUMMARY}

In this article we have reviewed the evidence for a twoprocess response to size and distance. The primary process involves the perception of size, distance, and (probably) perceived direction or changes in perceived direction. It is expressed by the SDIH, which, in agreement with the suggestion of McCready $(1965,1985)$, possibly should be expressed as $S^{\prime} / D^{\prime}=\tan \theta^{\prime}$. The secondary process is the result of off-sized perceptions, defined as $S_{\mathfrak{c}} / S^{\prime}$. The term $S^{\prime}$ is the perceived size made available to the observer by the stimulus present at the moment of observation. The term $S_{\mathrm{c}}$ is the remembered value of the object resulting from suggestion or from familiarity, including the remembered size of a prior presentation. When $S_{\mathrm{c}}$ differs from $S^{\prime}$, the immediate stimulus is perceived as an off-sized object. If it is perceived as a small off-sized object $\left(S_{\mathrm{c}}>S^{\prime}\right)$ or as a large off-sized object $\left(S_{\mathrm{c}}<S^{\prime}\right)$, it will tend to be reported as being at a greater or lesser distance $\left(D_{c}\right)$ than the distance at which it is perceived (Equation 2). In the primary process, $S^{\prime}$ and $D^{\prime}$ are always perceptual. In the secondary process, $S_{\mathrm{c}}$ is always cognitive. Whether $D_{c}$ is cognitive or perceptual is decided by a measure of perceived distance called the head motion procedure. The head motion procedure indirectly measures perceived distance using the apparent motion $\left(W^{\prime}\right)$ concomitant with the lateral motion of the head (Equation 9). The head motion procedure, unlike a direct response to distance, is considered to reflect only perceived, not cognitive, distance. Casual suggestion, familiarity, and optical expansion result in robust changes in direct reports of distance based on off-sized perceptions. But it was found, using the head motion procedure, that the effect of casual suggestion on distance reports was entirely cognitive (Gogel, 1981b) and that the effect of familiar size on distance reports was mainly but not entirely cognitive (Gogel, 1976), whereas optical expansion could produce completely perceptual modifications of distance (Swanston \& Gogel, 1986). The results from optical expansion, however, were not in agreement with Equation 1. Whether they are in agreement with Equation 15 remains to be determined.

Studies using the head motion procedure provide evidence that the effect of the secondary process on direct reports of distance can range from purely cognitive to purely perceptual. Whenever remembered size $\left(S_{c}\right)$, whether due to familiarity or suggestion, is the same as perceived size $\left(S^{\prime}\right)$, the $D_{\mathrm{c}}$ from the secondary process (Equation 2) and the $D^{\prime}$ from the primary process are in agreement. Whenever $S_{\mathrm{c}}$ and $S^{\prime}$ are different (an off-sized perception), the resulting $D_{\mathrm{c}}$ from the secondary process differs from the $D^{\prime}$ from the primary process. In this case, $D_{\mathrm{c}}$ and $D^{\prime}$, like $S_{\mathrm{c}}$ and $S^{\prime}$, disagree in the information they provide to direct responses, such as verbal reports of size $\left(S_{\mathrm{R}}\right)$ or distance $\left(D_{\mathrm{R}}\right)$. The resolution of this disagreement is reflected in the weights given primary and secondary processes in the weighting equations (Equations 3 and 4). These equations indicate the proportion $(a)$ of the total report of distance $\left(D_{\mathrm{R}}\right)$ determined by $D^{\prime}$, rather than by $D_{\mathrm{c}}$ (Equation 3), or the proportion $(b)$ of the total report of size $\left(S_{R}\right)$ determined by $S^{\prime}$, rather than by $S_{c}$ (Equation 4).

If $a$ and $b$ are the same for $D_{\mathrm{R}}$ as for $S_{\mathrm{R}}$, but are not unity (i.e., $D_{c}$ and $S_{c}$ contribute to the responses), the resulting relations between $D_{R}, S_{R}$, and the visual angle (Equation 7) will have the same form as the SDIH (Equation 1). This does not constitute an appropriate validation of the SDIH, however, since the SDIH involves only the primary process. If the weights $a$ and $b$ differ, $D_{\mathrm{R}}$ and $S_{R}$ will not satisfy Equation 1 . In this case, the data do not constitute an appropriate refutation of the SDIH, since the deviation from Equation 1 was produced by cognitive factors $\left(S_{\mathrm{c}}\right.$ and $\left.D_{\mathrm{c}}\right)$. Cognitive effects from off-sized judgments do not contribute to measures of perceived distance if the head motion procedure, rather than direct reports, is used. If a procedure similarly immune to $\operatorname{cog}$ nitive contributions were available for measuring perceived size, the combination of this procedure and the head motion procedure would provide a cognition-free test of the SDIH. Unfortunately, no procedures for measuring perceived size $\left(S^{\prime}\right)$ are available that are likely to totally avoid the effects of $S_{\mathrm{c}}$. A possible way of at least partially evaluating the contributions of $S_{c}$ to the direct response to size is to compare the results obtained from objective and apparent size instructions. In a study by Gogel and Da Silva (in press), observers' judgments of size, as measured by a tactile comparison adjustment, differed for the two kinds of instructions; however, the instructions had no significant effect on verbal reports of distance. Perhaps the distinction between $S_{\mathrm{c}}$ and $S^{\prime}$, since it concerns a memory and a present stimulation, is clear to the observer, whereas $D_{c}$, as shown by Equation 2, 
often involves a confluence of cognition and perception ( $S_{\mathrm{c}}$ as well as $D^{\prime}$ and $S^{\prime}$ ) and is not readily distinguished from $D^{\prime}$ by the observer.

Although $S_{\mathrm{c}}$ is an important variable in spatial responses, the characteristics of the internal representation that permit a memory to provide a standard for a current stimulus need to be specified. In some cases, such as that of a familiar object, similarities of detail and shape between the memory and the present stimulus are important. In the case of optical expansion, the temporal contiguity of the sequential presentation of sizes may permit some relaxation of the requirement that the shapes be similar. For example, it has been shown by Johansson (1964) that a two-dimensional optical change can produce apparent motion in depth, even if the rate of change is different horizontally and vertically, thereby producing changes in the form of the stimulus. Perceived object identity between successive moments of stimulation also can persist despite the robust transformations of physical form common to biological motions (Johansson, 1975). In addition, it seems that form changes can result in some continuity of identity even though no obviously identifiable invariance seems to be available in the physical transformations. For example, Ball, Ballot, and Dibble (1983) optically expanded a montage of shapes successively presented to infants and obtained a "looming" response. A somewhat similar example, occurring in a frontoparallel plane, is the apparent (phi) motion that can be seen between different stimulus forms, such as an arrow and a circle (Kolers \& Pomerantz, 1971). Contiguity (possibly spatial as well as temporal) seems to increase the ability of the nervous system to accept differing stimuli as instances of the same event. If the concept of changing size can occur despite substantial changes in shape, the role of off-sized perceptions in the production of depth responses, whether cognitive or perceptual, may be quite general.

In the case of optical expansion or contraction, Equation 1 clearly was unable to satisfy the obtained data. But if Equation 15, involving $\theta^{\prime}$ rather than $\theta$, is appropriate to represent the $\mathrm{SDIH}$, as has been suggested by McCready (1985), the SDIH might then be found to be consistent with the data obtained from the optical change. Unfortunately, some phenomena provided by McCready (1985) to justify Equation 15 can be explained in terms of cognitive factors involving off-sized perceptions (e.g., size-distance paradoxes). Although the errors in perceived size associated with geometrical illusions might be explained by differences between $\theta^{\prime}$ and $\theta$, this remains to be demonstrated. On the other hand, there is geometrical (Figure 4) and experimental evidence to suggest that the SDIH is an instance of the apparent distance/pivot distance hypothesis (Gogel \& Tietz, 1979; Gogel et al., 1985). If so, the importance of the perception of changes in direction in the latter hypothesis would indicate that it is equally important in the former hypothesis.
The physical variables of mass, length, and time are essential for the analysis of physical events and for the specification of higher order physical variables, such as force and momentum. It is suggested that in a similar way, the perceptual variables of size (or extent in a frontoparallel plane), distance, and direction are essential for the analysis of the perception of spatial events and for the specification of higher order perceptual variables, such as the perception of visual angle and of the pivot distance. Perceptual equations, such as Equations 10 and 15, express the interrelations between these basic perceptual variables. Such interrelations are found in the quantitative description of a wide range of phenomena, including a variety of invariance hypotheses (Gogel, 1984) and a variety of phenomena relevant to the adjacency principle (Fox \& Patterson, 1981; Gogel \& Mershon, 1977; Patterson \& Fox, 1983). The study of such perceptual interrelations requires the controlled manipulation of the basic perceptual variables $S^{\prime}$ and $D^{\prime}$, and, possibly, $\phi^{\prime}$ or $\theta^{\prime}$. The perception of $D^{\prime}$ independently of $D$ is readily manipulated by cues of distance, with $D^{\prime}$ in turn providing modifications of $S^{\prime}$ independently of $S$, as in the case of Emmert's Law (Weintraub \& Gardner, 1970). Unfortunately, the opportunities to manipulate $\theta^{\prime}$ or $\phi^{\prime}$ independently of $\theta$ or $\phi$ are more limited, although some variations can be produced as a result of the difference between the egocenter and the interocular axis (Ono, 1970), or perhaps by perceptual learning (Tietz \& Gogel, 1978).

\section{REFERENCES}

BAIRD, J. C. (1963). Retinal and assumed size cues as determinants of size and distance perception. Journal of Experimental Psychology, 66, 155-162.

Ball, W. A., Ballot, R., * Dibble, A. (1983). Stimulus dimensionality and infants' perceived movement in depth. Joumal of Genetic Psychology, 143, 193-200.

BiersDorf, W. R. (1966). Convergence and apparent distance as correlates of size judgments at near distances. Joumal of General Psychology, 75, 249-262.

CARLSON, V. R. (1960). Overestimation in size-constancy judgments. American Journal of Psychology, 73, 199-213.

CArLson, V. R. (1977). Instructions and perceptual constancy judgments. In W. Epstein (Ed.), Stability and constancy in visual perception: Mechanisms and processes. New York: Wiley.

Carlson, V. R., \& Tassone, E. P. (1962). A verbal measure of perspective attitude. American Journal of Psychology, 75, 644-647.

ColtheArT, M. (1969). The influence of haptic size information upon visual judgments of absolute distance. Perception \& Psychophysics, 5, 143-144.

Coltheart, M. (1970). The effect of verbal size information upon visual judgments of absolute distance. Perception \& Psychophysics, 9, 222-223.

EPSTEIN, W. (1965). Nonrelational judgments of size and distance. American Journal of Psychology, 78, 120-123.

Epstein, W., Park, J., \& Casey, A. (1961). The current status of the size-distance hypothesis. Psychological Bulletin, 58, 491-514.

ERIKsson, E. S., ZetTeraerg, P. (1975). Experience and veridical space perception (Report No. 169). Uppsala, Sweden: University of Uppsala, Department of Psychology.

Fitzpatrick, V., Pasnak, R., \& TYer, Z. E. (1982). The effect of familiar size at familiar distances. Perception, 11, 85-91. 
FolEY, J. M. (1965). Visual space: A scale of perceived relative direction. Proceedings of the 23rd Annual Convention of the American Psychological Association, 1, 49-50.

FoleY, J. M. (1968). Depth, size and distance in stereoscopic vision. Perception \& Psychophysics, 3, 265-274.

FoleY, J. M. (1972). The size-distance relation and intrinsic geometry of visual space: Implications for processing. Vision Research, 12, 323-332.

Foley, J. M. (1975). Error in visually directed manual pointing. Perception \& Psychophysics, 17, 69-74.

FOLEY, J. M. (1977) Effect of distance information and range on two indices of visually perceived distance. Perception, 6, 449-460.

FolEY, J. M., \& HELD, R. (1972). Visually directed pointing as a function of target distance, direction, and available cues. Perception \& Psychophysics, 12, 263-268.

Fox, R., \&ATterson, R. (1981). Depth separation and lateral interference. Perception \& Psychophysics, 30, 513-520.

Giusssky, A. S. (1951). Perceived size and distance in visual space. Psychological Review, 58, 460-482.

GiLLAM, B. J. (1968). Perception of slant when perspective and stereopsis conflict: Experiments with aniseikonic lenses. Joumal of Experimental Psychology, 78, 299-305.

GoGeL, W. C. (1965). Equidistance tendency and its consequences. Psychological Bulletin, 64, 153-163.

GoGEL, W. C. (1969a). The effect of object familiarity on the perception of size and distance. Quarterly Joumal of Experimental Psychology, 21, 239-247.

GoGEL, W. C. (1969b). The sensing of retinal size. Vision Research, 9, 1079-1094.

GOGEL, W. C. (1971). The validity of the size-distance invariance hypothesis with cue reduction. Perception \& Psychophysics, 9, 92-94.

GoGEL, W. C. (1972). Scalar perceptions with binocular cues of distance. American Journal of Psychology, 85, 477-498.

GoGel, W. C. (1974). Cognitive factors in spatial response. Psychologia, 17, 213-225.

GoGEL, W. C. (1976). An indirect method of measuring perceived distance from familiar size. Perception \& Psychophysics, 20, 419-429.

GOGEL, W. C. (1977). An indirect measure of perceived distance from oculomotor cues. Perception \& Psychophysics, 21, 3-11.

GoGEL, W. C. (1979). The common occurrence of errors of perceived distance. Perception \& Psychophysics, 25, 2-11.

Gogel, W. C. (1981a). Perceived depth is a necessary factor in apparent motion concomitant with head motion: A reply to Shebilske and Proffitt. Perception \& Psychophysics, 29, 173-177.

GoGEL, W. C. (1981b). The role of suggested size in distance responses. Perception \& Psychophysics, 30, 149-155.

GoGEL, W. C. (1982). Analysis of the perception of motion concomitant with a lateral motion of the head. Perception \& Psychophysics, 32, 241-250.

GoGEL, W. C. (1984). The role of perceptual interrelations in figural synthesis. In P. C. Dodwell \& T. Caelli (Eds.), Figural synthesis (pp. 31-82). Hillsdale, NJ: Erlbaum.

Gogel, W. C., DA Silva, J. A. (in press). Familiar size and the theory of off-sized perceptions. Perception \& Psychophysics.

Gogel, W. C., Hartman, B. O., \& Harker, G. S. (1957). The retinal size of a familiar object as a determiner of apparent distance. Psychological Monographs, 71(13, Whole No. 442).

Gogel, W. C., Loomis, J. M., Newman, N. J., \& Sharkey, T. J. (1985). Agreement between indirect measures of perceived distance. Perception \& Psychophysics, 37, 17-27.

Gogel, W. C., MershoN, D. H. (1977). Local autonomy in visual space. Scandinavian Joumal of Psychology, 18, 237-250.

Gogel, W. C., Mertens, H. W. (1967). Perceived size and distance of familiar objects. Perceptual \& Motor Skills, 25, 213-225.

Gogel, W. C., \& Mertens, H. W. (1968). Perceived depth between familiar objects. Joumal of Experimental Psychology, 77, 206-211.

GoGel, W. C., Newton, R. E. (1969). Perception of off-sized objects. Perception \& Psychophysics, 5, 7-9.

GoGEL, W. C., \& STURM, R. D. (1971). Directional separation and the size cue to distance. Psychologische Forschung, 35, 57-80.
Gogel, W. C., \& Tietz, J. D. (1973). Absolute motion parallax and the specific distance tendency. Perception \& Psychophysics, 13, 284-292.

Gogel, W. C., \& Tietz, J. D. (1977). Eye fixation and attention as modifiers of perceived distance. Perceptual \& Motor Skills, 45, 343-362.

GoGEL, W. C., \& TIETz, J. D. (1979). A comparison of oculomotor and motion parallax cues of egocentric distance. Vision Research, 19, 1161-1170.

GrUBer, H. E. (1954). The relation of perceived size to perceived distance. American Joumal of Psychology, 67, 411-426.

HAstoRF, A. H. (1950). The influence of suggestion on the relationship between stimulus size and perceived distance. Journal of Psychology, 29, 195-217.

Heineman, E. G., Tulving, E., Nachmis, J. (1959). The effect of oculomotor adjustments on apparent size. American Joumal of Psychology, 72, 32-45.

Hennessy, R. T., Iida, T., Shinna, K., \& Leibowitz, H. W. (1976). The effect of pupil size on accommodation. Vision Research, 16, 587-589.

Hignshiyama, A. (1977). Perceived size and distance as a perceptual conflict between two processing modes. Perception \& Psychophysics, 22, 206-211.

Higashiyama, A. (1979). The perception of size and distance under monocular observation. Perception \& Psychophysics, 26, $230-234$.

HigashiYAma, A. (1983). A variety of size and distance judgments under monocular observation: Instruction and individual differences. $\mathrm{Hu}$ man Science (The University of Asaka Prefecture, The Human Sciences Society), 13-14, 91-111.

ItTELsON, W. H. (1951a). Size as a cue to distance: Radial motion. American Journal of Psychology, 64, 188-202.

ITtelson, W. H. (1951b). Size as a cue to distance: Static localization. American Journal of Psychology, 64, 54-67.

Johansson, G. (1964). Perception of motion and changing form. Scandinavian Journal of Psychology, 5, 181-208.

Johansson, G. (1975). Visual motion perception. Scientific American, 232, No. 6, 76-88.

Kaufman, L., Rock, I. (1962). The moon illusion: II. Science, 136, 1023-1031.

Kilpatrick, F. P., \& ItTelson, W. H. (1953). The size-distance invariance hypothesis. Psychological Review, 60, 223-231.

Kolers, P. A., Pomerantz, J. R. (1971). Figural change in apparent motion. Journal of Experimental Psychology, 87, 99-108.

KомоDA, М. К. (1970). The nature of accommodation-convergence micropsia. Unpublished doctoral dissertation, York University, Toronto, Ontario, Canada.

KomodA, M. K., ONo, H. (1974). Oculomotor adjustments and sizedistance perception. Perception \& Psychophysics, 15, 353-360.

MacCracken, P. J., Gogel, W. C., Blum, G. S. (1980). Effects of posthypnotic suggestion on perceived egocentric distance. Perception, 9, 561-568.

MCCready, D. W., JR. (1965). Size-distance perception and accommodation-convergence micropsia: A critique. Vision Research, 5, 189-206.

MCCREADY, D. (1985). On size, distance, and visual angle perception. Perception \& Psychophysics, 37, 323-334.

McCrendy, D. (1986). Moon illusions redescribed. Perception \& Psychophysics, 39, 64-72.

Mershon, D. H., Kennedy, M., Falacara, G. (1977). On the use of calibration equations in perception research. Perception, 6, 299-311.

Mershon, D. H., LemBo, V. L. (1977). Scalar perceptions of distance in simple binocular configurations. American Journal of Psychology, 90, 17-28.

ONo, H. (1970). Some thoughts on different perceptual tasks related to size and distance. In J. C. Baird (Ed.), Human Space Perception: Proceedings of the Dartmouth Conference. Psychonomic Monograph Supplements, 3(13, Whole No. 45).

ONo, H., MuTER, P., \& Mrrson, L. (1974). Size-distance paradox with accommodation micropsia. Perception \& Psychophysics, 15, 301-307. OWENS, D. A., * LeIBowitZ, H. W. (1976). Oculomotor adjustments 
in darkness and the specific distance tendency. Perception \& Psychophysics, 20, 2-9.

Owens, D. A., Leibowitz, H. W. (1983). Perceptual and motor consequences of tonic vergence. In C. M. Schor \& K. J. Ciuffreda (Eds.), Vergence eye movements: Basic and clinical aspects. Boston: Butterworth.

Park, J. N., Michaelson, G. J. (1974). Distance judgments under different size-information conditions. Perception \& Psychophysics, $15,57-60$.

Pattreson, R., Fox, R. (1983). Depth separation and the Ponzo illusion. Perception \& Psychophysics, 34, 25-28.

Predebon, G. M., Wenderoth, P. M., * Curthoys, I. S. (1974). The effects of instructions and distance on judgments of off-size familiar objects and normal viewing conditions. American Journal of Psychology, 87, 425-439.

Rock, I., Hill, A. L., \& Fineman, M. (1968). Speed constancy as a function of size constancy. Perception \& Psychophysics, 4, 37-40.

Schlosiurg, H. (1950). A note on depth perception, size constancy, and related topics. Psychological Review, 57, 314-317.

Swanston, M. T., Gogel, W. C. (1986). Perceived size and mo- tion in depth from optical expansion. Perception \& Psychophysics, 39, 309-326.

TieTZ, J. D., GoGel, W. C. (1978). Adaptation to apparent concomitant motion in the absence of physical or retinal motion. Bulletin of the Psychonomic Society, 12, 1-4.

Weintraub, D. J., \& Gardner, G. T. (1970). Emmert's laws: Size constancy versus optical geometry. American Joumal of Psychology, $83,40-54$.

Wist, E. R., Diener, H. C., Dichgans, J. (1976). Motion constancy dependent upon perceived distance and the spatial frequency of the stimulus pattern. Perception \& Psychophysics, 19, 485-491.

\section{NOTE}

1. Throughout this article the term tangent of the angle is used as a convenient approximation of the more accurate term twice the tangent of half the angle.

(Manuscript received November 7, 1983; revision accepted for publication October $30,1986$. 